\title{
Diffusiophoresis and electrophoresis of a charged sphere parallel to one or two plane walls
}

\author{
Po Y. Chen, Huan J. Keh * \\ Department of Chemical Engineering, National Taiwan University, Taipei 106-17, Taiwan, Republic of China
}

Received 7 September 2004; accepted 20 January 2005

Available online 2 March 2005

\begin{abstract}
The diffusiophoretic and electrophoretic motions of a dielectric spherical particle in an electrolyte solution located between two infinite parallel plane walls are studied theoretically. The imposed electrolyte concentration gradient or electric field is constant and parallel to the two plates, which may be either impermeable to the ions/charges or prescribed with the far-field concentration/potential distribution. The electrical double layer at the particle surface is assumed to be thin relative to the particle radius and to the particle-wall gap widths, but the polarization effect of the mobile ions in the diffuse layer is incorporated. The presence of the neighboring walls causes two basic effects on the particle velocity: first, the local electrolyte concentration gradient or electric field on the particle surface is enhanced or reduced by the walls, thereby speeding up or slowing down the particle; second, the walls increase the viscous retardation of the moving particle. To solve the conservative equations, the general solution is constructed from the fundamental solutions in both rectangular and spherical coordinates. The boundary conditions are enforced first at the plane walls by the Fourier transforms and then on the particle surface by a collocation technique. Numerical results for the diffusiophoretic and electrophoretic velocities of the particle relative to those of a particle under identical conditions in an unbounded solution are presented for various values of the relevant parameters including the relative separation distances between the particle and the two plates. For the special case of motions of a spherical particle parallel to a single plate and in the central plane of a slit, the collocation results agree well with the approximate analytical solutions obtained by using a method of reflections. The presence of the lateral walls can reduce or enhance the particle velocity, depending on the properties of the particle-solution system, the relative particlewall separation distances, and the electrochemical boundary condition at the walls. In general, the boundary effects on diffusiophoresis and electrophoresis are quite significant and complicated, and they no longer vary monotonically with the separation distances for some situations. (c) 2005 Elsevier Inc. All rights reserved.
\end{abstract}

Keywords: Diffusiophoresis; Electrophoresis; Boundary effects; Thin but polarized double layer

\section{Introduction}

The transport behavior of small particles in a continuous medium at low Reynolds number is of much fundamental and practical interest. In general, driving forces for motions of colloidal particles include concentration gradients of the particles themselves (diffusion), bulk velocities of the disperse medium (convection), and gravitational fields (sedimentation). Problems of the colloidal transport induced by these well-known driving forces have been treated exten-

\footnotetext{
* Corresponding author.

E-mail address: huan@ntu.edu.tw (H.J. Keh).
}

sively in the past. Another category of driving forces for the motions of colloidal particles involves a nonuniform imposed field (such as electric potential, temperature, or solute concentration) that interacts with the surface of each particle. The particle motions associated with this mechanism, known as "phoretic motions," have also received a considerable amount of attention recently [1-4].

Perhaps the most familiar example of phoretic motion is electrophoresis, which results from the interaction between an external electric field and the electric double layer surrounding a charged particle and is widely used for particle characterization and separation in a variety of colloidal and biological systems [5-7]. The electrophoretic veloc- 
ity $\mathbf{U}^{(0)}$ of a single dielectric particle suspended in an unbounded electrolyte solution is simply related to the uniformly applied electric field $\mathbf{E}^{\infty}$ by the Smoluchowski equation [8-10],

$\mathbf{U}^{(0)}=\frac{\varepsilon \zeta}{4 \pi \eta} \mathbf{E}^{\infty}$.

Here, $\varepsilon / 4 \pi$ is the fluid permittivity, $\eta$ is the fluid viscosity, and $\zeta$ is the zeta potential of the particle surface.

Another example of phoretic motions is diffusiophoresis, which is the migration of a particle in response to the macroscopic concentration gradient of a solute and can be applied to certain latex-particle coating processes $[1,11]$. The particle moves toward or away from a region of higher solute concentration, depending on long-range interactions between the solute molecules and the particle. In an unbounded solution of a symmetric electrolyte with a constant concentration gradient $\nabla n^{\infty}$, the diffusiophoretic velocity of a charged particle is $[12,13]$

$\mathbf{U}^{(0)}=\frac{\varepsilon \zeta}{4 \pi \eta} \frac{k T}{Z e} \frac{\nabla n^{\infty}}{n^{\infty}(\mathbf{0})}\left(\alpha+\bar{\zeta}^{-1} \ln \cosh \bar{\zeta}\right)$,

with the dimensionless parameters

$\alpha=\frac{D_{2}-D_{1}}{D_{2}+D_{1}}$,

$\bar{\zeta}=\frac{Z e \zeta}{4 k T}$.

Here, $n^{\infty}(\mathbf{0})$ is the macroscopic electrolyte concentration measured at the particle center $\mathbf{0}$ in the absence of the particle, $D_{1}$ and $D_{2}$ are the diffusion coefficients of the anion and cation, respectively, $Z$ is the absolute value of the valences of the ions, $e$ is the charge of a proton, $k$ is the Boltzmann constant, and $T$ is the absolute temperature. For the special case $D_{2}=D_{1}$ or $\alpha=0$, Eq. (2) predicts that the particle movement (due to chemiphoresis only) is in the direction of increasing electrolyte concentration regardless of the sign of $\zeta$ and the particle velocity is a monotonic increasing function of the magnitude of $\zeta$.

Equations (1) and (2) indicate that the electrophoretic and diffusiophoretic velocities of a dielectric particle having a uniform zeta potential on its surface are independent of the particle size and shape (and there is no rotational motion of the particle). However, their validity is based on the assumptions that the local radii of curvature of the particle are much larger than the thickness of the electric double layer at the particle surface and that the effect of polarization (relaxation effect) of the diffuse ions in the double layer due to nonuniform "osmotic" flow is negligible. In fact, important advances were made in the past in the evaluation of the phoretic velocities of colloidal particles relaxing these assumptions.

Taking the double-layer distortion from equilibrium as a perturbation, O'Brien and White [14] obtained a numerical calculation for the electrophoretic velocity of a dielectric sphere of radius $a$ in a $\mathrm{KCl}$ solution which was applicable to arbitrary values of $\zeta$ and $\kappa a$, where $\kappa^{-1}$ is the Debye screening length equal to $\left(8 \pi Z^{2} e^{2} n^{\infty} / \varepsilon k T\right)^{1 / 2}$. On the other hand, Dukhin and Derjaguin [1] obtained an analytical expression for the electrophoretic mobility of a spherical particle surrounded by a thin but polarized double layer in the solution of a symmetric electrolyte. Later, O'Brien [15] generalized this analysis to the case of electrophoretic motion of a charged sphere in the solution containing an arbitrary combination of electrolytes. The essence of this thin-layer polarization approach is that a thin diffuse layer can still transport a significant amount of electrolyte ions in such a way as to affect the ionic transport outside the diffuse layer. The result for the electrophoretic velocity of a dielectric sphere with a thin but polarized double layer in a symmetric electrolyte solution can be expressed as [16]

$\mathbf{U}_{0}=\frac{\varepsilon \zeta}{12 \pi \eta} \mathbf{E}^{\infty}\left[2+c_{1}+c_{2}+\left(c_{1}-c_{2}\right) \bar{\zeta}^{-1} \ln \cosh \bar{\zeta}\right]$,

where coefficients $c_{1}$ and $c_{2}$ are defined by Eqs. (A.6a) and (A.6b). A comparison of Eq. (5) with the numerical results for the $\mathrm{KCl}$ solution [14] shows that the thin-layer polarization model is quite good over a wide range of zeta potentials when $\kappa a>20$. If $|\zeta|$ is small and $\kappa a$ is large, the interaction between the diffuse counterions and the particle surface is weak and the polarization of the double layer is also weak. In the limit of

$(\kappa a)^{-1} \exp (2|\bar{\zeta}|) \rightarrow 0$,

$c_{1}=c_{2}=1 / 2$ and Eq. (5) reduces to the Smoluchowski equation (1). In general, the electrophoretic velocity given by Eq. (5) is not a monotonic function of $\bar{\zeta}$ for a finite value of $\kappa a$, unlike the prediction of Eq. (1).

In contrast, Prieve and Roman [17] obtained a numerical solution for the diffusiophoretic velocity over a broad range of $\bar{\zeta}$ and $\kappa a$ for a charged sphere in concentration gradients of symmetric electrolytes $(\mathrm{KCl}$ or $\mathrm{NaCl})$ using the method of O'Brien and White [14]. On the other hand, analytical expressions for the velocity of a dielectric sphere with a thin but polarized double layer undergoing diffusiophoresis in electrolyte solutions have also been derived $[4,18]$. The result for this diffusiophoretic velocity in a symmetric electrolyte solution is

$$
\begin{aligned}
\mathbf{U}_{0}= & \frac{\varepsilon \zeta}{12 \pi \eta} \frac{k T}{Z e} \frac{\nabla n^{\infty}}{n^{\infty}(\mathbf{0})}\left\{c_{1}^{\prime}-c_{2}^{\prime}+\alpha\left(2+c_{1}+c_{2}\right)\right. \\
& \left.+\left[2+c_{1}^{\prime}+c_{2}^{\prime}+\alpha\left(c_{1}-c_{2}\right)\right] \bar{\zeta}^{-1} \ln \cosh \bar{\zeta}\right\},
\end{aligned}
$$

where coefficients $c_{1}^{\prime}$ and $c_{2}^{\prime}$ are defined by Eqs. (A.6c) and (A.6d). When $\kappa a>20$, the agreement between Eq. (7) and the numerical solution [17] is excellent for all reasonable values of the zeta potential. In the limiting situation given by Eq. (6), the effect of double-layer polarization disappears, $c_{1}=c_{2}=c_{1}^{\prime}=c_{2}^{\prime}=1 / 2$, and Eq. (7) reduces to Eq. (2). Even for the case of $D_{2}=D_{1}$, the particle velocity given by Eq. (7) for a finite value of $\kappa a$ may not be a monotonic function of the magnitude of $\bar{\zeta}$ and its direction can reverse 
(toward lower electrolyte concentration) when $|\bar{\zeta}|$ becomes large.

It could be found from Eqs. (5) and (7) that the effect of polarization of the diffuse layer is to decrease the particle velocity. The reason for this outcome is that the transport of the diffuse ions within the double layer reduces the local electrolyte gradient or electric field along the particle surface. Numerical calculations of Eqs. (5) and (7) show that, even when $\kappa a$ is as large as 300 , the effect of ionic transport inside the diffuse layer cannot be ignored if $|\zeta|$ equals several $k T / e$.

In practical applications of diffusiophoresis and electrophoresis, such as transport in channels of microfluidic devices [19-22], particles are not isolated and the surrounding fluid is externally bounded by solid walls. Thus, it is important to determine if the presence of neighboring boundaries significantly affects the movement of particles. In the limiting case where Eqs. (1) and (2) are applicable, the normalized velocity field of the immense fluid that is dragged by a particle during diffusiophoresis is the same as for the electrophoresis of the particle [2]; thus, the boundary effects on electrophoresis under the situation of an infinitesimally thin double layer [satisfying Eq. (6)], which have been studied extensively in the past [23-29], can be taken to interpret those in diffusiophoresis.

When the polarization effect of diffuse ions in the double layer surrounding the particle is considered, the boundary effects on diffusiophoresis can be quite different from those on electrophoresis. Through the use of a boundary collocation technique, the diffusiophoretic and electrophoretic motions of a colloidal sphere with a thin but polarized diffuse layer perpendicular to a plane wall were examined [30]. The wall effect on each transport mechanism was found to be a complicated function of the properties of the particle and ions. In this work we present a theoretical investigation of the diffusiophoretic and electrophoretic motions of a charged sphere with a thin but polarized double layer parallel to a single plane wall and to two plane walls at an arbitrary position between them. The quasi-steady equations of conservation applicable to each system are solved by using both the boundary collocation technique numerically and a method of reflections analytically. The exact numerical solutions for the particle velocities are obtained with good convergence for various cases and agree well with the approximate analytical solutions. In the limiting case of Eq. (6), our results are in excellent agreement with those available in the literature for electrophoresis [23,24].

\section{Analysis for diffusiophoresis}

In this section we consider the quasi-steady diffusiophoretic motion of a dielectric spherical particle of radius $a$ in a solution of a symmetrically charged electrolyte parallel to two infinite plane walls whose distances from the center of the particle are $b$ and $c$, as shown in Fig. 1. Here

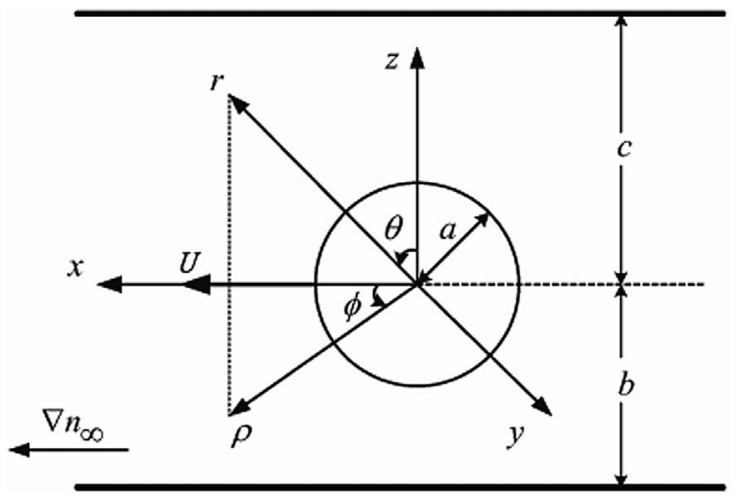

Fig. 1. Geometrical sketch for the diffusiophoresis of a spherical particle parallel to two plane walls at an arbitrary position between them.

$(x, y, z),(\rho, \phi, z)$, and $(r, \theta, \phi)$ denote the rectangular, circular cylindrical, and spherical coordinate systems, respectively, and the origin of coordinates is chosen at the particle center. A linear electrolyte concentration field $n^{\infty}(x)$ with a uniform gradient in the $x$ direction is imposed in the surrounding fluid far away from the particle. The particle is charged uniformly on the surface, and the thickness of the electrical double layer is assumed to be small in comparison with the radius of the particle and the spacing between the particle and each wall. Hence, the fluid phase can be divided into two regions: an "inner" region defined as the thin double layer adjacent to each solid surface and an "outer" region defined as the remainder of the fluid, which is electrically neutral. The objective is to determine the correction to Eq. (7) for the particle velocity due to the presence of the plane walls.

Before the diffusiophoretic velocity of the particle is determined, the electrochemical potential and velocity fields in the fluid phase need to be found.

\subsection{Electrochemical potential distribution}

The Peclet number of the system is assumed to be small. Hence, the equation of conservation of each ionic species for the outer region of the fluid solution is the Laplace equation $[4,15]$,

$\nabla^{2} \mu_{m}=0, \quad m=1,2$.

In Eq. (8), $\mu_{m}$ is the electrochemical potential energy of ionic species $m$, defined by

$\mu_{m}=\mu_{m}^{0}+k T \ln n_{m}+z_{m} e \Phi$,

where $\mu_{m}^{0}$ is a constant, $n_{m}$ and $z_{m}$ are the concentration and valence, respectively, of type $m$ ions, and $\Phi$ is the electric potential. $m$ equal to 1 and 2 refers to the anion and cation, respectively, so $-z_{1}=z_{2}=Z>0$. Note that, in the outer region, $n_{1}=n_{2}=n$, and both $n$ and $\Phi$ also satisfy Laplace's equation.

Equation (8) satisfies the boundary condition at the particle "surface" (outer limit of the thin double layer) obtained 
by solving for the electrochemical potentials in the inner region and using a matching procedure to ensure a continuous solution in the whole fluid phase $[4,15]$,

$r=a^{+}: \quad \mathbf{e}_{r} \cdot \nabla \mu_{m}=-\sum_{i=1}^{2} \beta_{m i} \mathbf{I}: \nabla_{\mathrm{s}} \nabla_{\mathrm{s}} \mu_{i}, \quad m=1,2$,

where the relaxation coefficients are

$$
\begin{aligned}
\beta_{11}= & \frac{1}{\kappa}\left[4\left(1+\frac{3 f_{1}}{Z^{2}}\right) \exp (\bar{\zeta}) \sinh \bar{\zeta}\right. \\
& \left.-\frac{12 f_{1}}{Z^{2}}(\bar{\zeta}+\ln \cosh \bar{\zeta})\right], \\
\beta_{12}= & -\frac{1}{\kappa}\left(\frac{12 f_{1}}{Z^{2}}\right) \ln \cosh \bar{\zeta}, \\
\beta_{21}= & -\frac{1}{\kappa}\left(\frac{12 f_{2}}{Z^{2}}\right) \ln \cosh \bar{\zeta}, \\
\beta_{22}= & \frac{1}{\kappa}\left[-4\left(1+\frac{3 f_{2}}{Z^{2}}\right) \exp (-\bar{\zeta}) \sinh \bar{\zeta}\right. \\
& \left.+\frac{12 f_{2}}{Z^{2}}(\bar{\zeta}-\ln \cosh \bar{\zeta})\right] .
\end{aligned}
$$

In the above equation, $f_{m}=\varepsilon k^{2} T^{2} / 6 \pi \eta e^{2} D_{m}, \bar{\zeta}$ was defined by Eq. (4), $\mathbf{e}_{r}$ is the unit vector in the direction of $r$, $\mathbf{I}$ is the unit dyadic, $\nabla_{\mathrm{s}}=\left(\mathbf{I}-\mathbf{e}_{r} \mathbf{e}_{r}\right) \cdot \nabla$ denotes the gradient operator along the particle surface, and $\mathbf{U}$ and $\boldsymbol{\Omega}$ are the instantaneous translational and angular velocities, respectively, of the particle to be determined. To obtain Eqs. (10) and (11), it was assumed that the concentration of each ionic species within the electric double layer is related to the electric potential energy by a Boltzmann distribution. Evidently, in the limit of Eq. (6), $\beta_{11}=\beta_{12}=\beta_{21}=\beta_{22}=0$.

The ionic electrochemical potentials far away from the particle approach the undisturbed values. Thus,

$z=c,-b: \quad \frac{\partial \mu_{m}}{\partial z}=0$,

$\rho \rightarrow \infty: \quad \mu_{m} \rightarrow \mu_{m_{\infty}}$,

where

$\mu_{m \infty}=\mu_{m}^{0}+k T\left[1-(-1)^{m} \alpha\right] \ln n^{\infty}$

and $\alpha$ was defined by Eq. (3). The second term in the brackets of Eq. (14) represents the contribution from the macroscopic electric field induced by the difference of ion diffusion rates [13,31]. Note that the boundary conditions given by Eq. (12) apply for the case of two impermeable and nonconducting plane walls (which can appear in practice) with a negligible relaxation effect in their thin interfacial diffuse layers. For the case of diffusiophoretic motion of a particle parallel to two plane walls prescribed with linear electrochemical potential profiles consistent with the far-field distributions (which is less practical), Eq. (12) should be replaced by

$z=c,-b: \quad \mu_{m}=\mu_{m \infty}$.

Since the governing equation and boundary conditions are linear, one can express the electrochemical potential distribution $\mu_{m}$, which is symmetric with respect to $y$ and antisymmetric with respect to $x$, as the superposition

$\mu_{m}=\mu_{m \mathrm{w}}+\mu_{m \mathrm{p}}, \quad m=1,2$.

Here, $\mu_{m w}$ is a double Fourier integral solution of Eq. (8) in rectangular coordinates that represents the disturbance produced by the plane walls plus the undisturbed field and is given by

$$
\begin{aligned}
\mu_{m \mathrm{w}}= & \mu_{m \infty}+\int_{0}^{\infty} \int_{0}^{\infty}\left(X_{m} e^{\kappa z}+Y_{m} e^{-\kappa z}\right) \\
& \times \sin (\hat{\alpha} x) \cos (\hat{\beta} y) \mathrm{d} \hat{\alpha} \mathrm{d} \hat{\beta},
\end{aligned}
$$

where $X_{m}$ and $Y_{m}$ are unknown functions of separation variables $\hat{\alpha}$ and $\hat{\beta}$, and $\kappa=\left(\hat{\alpha}^{2}+\hat{\beta}^{2}\right)^{1 / 2}$. The second term on the right-hand side of Eq. (16), $\mu_{m p}$, is a solution of Eq. (8) in spherical coordinates representing the disturbance generated by the spherical particle and is given by an infinite series in harmonics,

$\mu_{m \mathrm{p}}=\sum_{n=1}^{\infty} R_{m n} r^{-n-1} P_{n}^{1}(\mu) \cos \phi$,

where $P_{n}^{1}$ is the associated Legendre function of order $n$ and degree one, $\mu$ is used to denote $\cos \theta$ for brevity, and $R_{m n}$ are unknown constants. Note that a solution for $\mu_{m}$ of the form given by Eqs. (16)-(18) immediately satisfies the boundary condition at infinity in Eq. (13).

Substituting the electrochemical potential distribution $\mu_{m}$ given by Eqs. (16)-(18) into the boundary conditions in Eq. (12) or (15) and applying the Fourier sine and cosine transforms on the variables $x$ and $y$, respectively, lead to a solution for the functions $X_{m}$ and $Y_{m}$ in terms of the coefficients $R_{m n}$. After the substitution of this solution into Eq. (17) and utilization of the integral representations of the modified Bessel functions of the second kind, the distribution $\mu_{m}$ can be expressed as

$$
\mu_{m}=\mu_{m \infty}+\sum_{n=1}^{\infty} R_{m n} \delta_{n}^{(1)}(r, \mu) \cos \phi, \quad m=1,2,
$$

and the function $\delta_{n}^{(1)}(r, \mu)$ is defined by Eq. (B.1) in Appendix B.

Applying the boundary condition given by Eq. (10) to Eq. (19) yields

$$
\begin{aligned}
\sum_{n=1}^{\infty} \sum_{m=1}^{2} R_{m n} R_{m}^{\prime}(\mu)= & k T\left[\left(1-\frac{2 \beta_{11}}{a}\right)(1+\alpha)\right. \\
& \left.-\frac{2 \beta_{12}}{a}(1-\alpha)\right]\left(1-\mu^{2}\right)^{1 / 2}
\end{aligned}
$$




$$
\begin{aligned}
\sum_{n=1}^{\infty} \sum_{m=1}^{2} R_{m n} R_{m}^{\prime \prime}(\mu)= & k T\left[\left(1-\frac{2 \beta_{22}}{a}\right)(1-\alpha)\right. \\
& \left.-\frac{2 \beta_{21}}{a}(1+\alpha)\right]\left(1-\mu^{2}\right)^{1 / 2}
\end{aligned}
$$

where

$R_{1}^{\prime}(\mu)=\left(2 \beta_{11}-a\right) \delta_{n}^{(2)}(a, \mu)+a \beta_{11} \delta_{n}^{(4)}(a, \mu)$,

$R_{2}^{\prime}(\mu)=\beta_{12}\left[2 \delta_{n}^{(2)}(a, \mu)+a \delta_{n}^{(4)}(a, \mu)\right]$,

$R_{1}^{\prime \prime}(\mu)=\beta_{21}\left[2 \delta_{n}^{(2)}(a, \mu)+a \delta_{n}^{(4)}(a, \mu)\right]$,

$R_{2}^{\prime \prime}(\mu)=\left(2 \beta_{22}-a\right) \delta_{n}^{(2)}(a, \mu)+a \beta_{22} \delta_{n}^{(4)}(a, \mu)$,

and the definitions of functions $\delta_{n}^{(2)}(r, \mu)$ and $\delta_{n}^{(4)}(r, \mu)$ are given by Eqs. (B.2) and (B.4). Note that the dependence of $\phi$ factors out in Eq. (20) and the definite integrals in $\delta_{n}^{(1)}, \delta_{n}^{(2)}$, and $\delta_{n}^{(4)}$ must be performed numerically.

To satisfy the condition in Eq. (20) exactly along the entire surface of the particle would require the solution of the entire infinite array of unknown constants $R_{m n}$. However, the collocation method [30,32] enforces the boundary condition at a finite number of discrete points on the halfcircular generating arc of the sphere (from $\theta=0$ to $\theta=\pi$ ) and truncates the infinite series in Eq. (19) into finite ones. If the spherical boundary is approximated by satisfying the condition of Eq. (10) at $M$ discrete points on its generating arc, the infinite series in Eq. (19) is truncated after $M$ terms, resulting in a system of $2 M$ simultaneous linear algebraic equations in the truncated form of Eq. (20). This matrix equation can be numerically solved to yield the $2 M$ unknown constants $R_{m n}$ required in the truncated form of Eq. (19) for the electrochemical potential distribution. The accuracy of the boundary-collocation/truncation technique can be improved to any degree by taking a sufficiently large value of $M$. Naturally, as $M \rightarrow \infty$, the truncation error vanishes and the overall accuracy of the solution depends only on the numerical integration required in evaluating the matrix elements.

\subsection{Fluid velocity distribution}

With knowledge of the solution for the ionic electrochemical potential distribution on the particle surface which drives the migration, we can now proceed to find the flow field. The fluid solution is assumed to be incompressible and Newtonian. Owing to the low Reynolds number, the fluid motion in the outer region caused by the diffusiophoretic motion of the particle is governed by the Stokes equations,

$\eta \nabla^{2} \mathbf{v}-\nabla p=\mathbf{0}$

$\nabla \cdot \mathbf{v}=0$,

where $\mathbf{v}$ is the velocity field for the fluid flow and $p$ is the dynamic pressure distribution.
The boundary conditions for the fluid velocity at the particle surface $[4,15]$, on the plane walls, and far removed from the particle are

$r=a^{+}: \quad \mathbf{v}=\mathbf{v}_{\mathrm{s}} \equiv \mathbf{U}+\boldsymbol{\Omega} \times \mathbf{r}+\mathbf{v}^{(\mathrm{s})}$,

$z=c,-b: \quad \mathbf{v}=\mathbf{0}$,

$\rho \rightarrow \infty: \quad \mathbf{v}=\mathbf{0}$.

In Eq. (23), the apparent slip velocity due to diffusioosmotic effect is

$\mathbf{v}^{(\mathrm{s})}=\sum_{m=1}^{2} W_{m} \nabla_{\mathrm{s}} \mu_{m}$

where

$W_{m}=\frac{\varepsilon}{2 \pi \eta} \frac{k T}{(Z e)^{2}}\left[-(-1)^{m} \bar{\zeta}+\ln \cosh \bar{\zeta}\right]$,

and $\mathbf{U}=U \mathbf{e}_{x}$ and $\boldsymbol{\Omega}=\Omega \mathbf{e}_{y}$ are the translational and angular velocities of the particle undergoing diffusiophoresis, to be determined. For the asymmetric problem, $b \neq c$, the assumption that the sphere would migrate in a direction parallel to the solute concentration gradient is justified in the absence of fluid inertia. Note that the possible osmotic flow caused by the plane walls is ignored for simplicity.

A fundamental solution to Eq. (22) which satisfies Eqs. (24) and (25) can be obtained in a way similar to that for Eq. (19), with the form

$\mathbf{v}=v_{x} \mathbf{e}_{x}+v_{y} \mathbf{e}_{y}+v_{z} \mathbf{e}_{z}$,

where

$v_{x}=\sum_{n=1}^{\infty}\left[A_{n}\left(A_{n}^{\prime}+\alpha_{n}^{\prime}\right)+B_{n}\left(B_{n}^{\prime}+\beta_{n}^{\prime}\right)+C_{n}\left(C_{n}^{\prime}+\gamma_{n}^{\prime}\right)\right]$,

$v_{y}=\sum_{n=1}^{\infty}\left[A_{n}\left(A_{n}^{\prime \prime}+\alpha_{n}^{\prime \prime}\right)+B_{n}\left(B_{n}^{\prime \prime}+\beta_{n}^{\prime \prime}\right)+C_{n}\left(C_{n}^{\prime \prime}+\gamma_{n}^{\prime \prime}\right)\right]$,

$v_{z}=\sum_{n=1}^{\infty}\left[A_{n}\left(A_{n}^{\prime \prime \prime}+\alpha_{n}^{\prime \prime \prime}\right)+B_{n}\left(B_{n}^{\prime \prime \prime}+\beta_{n}^{\prime \prime \prime}\right)+C_{n}\left(C_{n}^{\prime \prime \prime}+\gamma_{n}^{\prime \prime \prime}\right)\right]$

Here, the primed $A_{n}, B_{n}, C_{n}, \alpha_{n}, \beta_{n}$, and $\gamma_{n}$ are functions of position involving associated Legendre functions of $\mu$ or $\cos \theta$ defined by Eq. (2.6) and in the form of integration (which need to be performed numerically) defined by Eq. (C.1) of Ganatos et al. [32], and $A_{n}, B_{n}$, and $C_{n}$ are unknown constants.

The boundary condition that remains to be satisfied is that on the particle surface. Substituting Eqs. (19) and (28) into Eq. (23), one obtains 


$$
\begin{aligned}
& \sum_{n=1}^{\infty}\left[A_{n}\left(A_{n}^{\prime}+\alpha_{n}^{\prime}\right)+B_{n}\left(B_{n}^{\prime}+\beta_{n}^{\prime}\right)+C_{n}\left(C_{n}^{\prime}+\gamma_{n}^{\prime}\right)\right]_{r=a} \\
& =U+a \Omega \mu \\
& -\sum_{m=1}^{2} W_{m}\left[H_{1 m}(\mu) \mu \cos ^{2} \phi+H_{2 m}(\mu) \sin ^{2} \phi\right], \\
& \sum_{n=1}^{\infty}\left[A_{n}\left(A_{n}^{\prime \prime}+\alpha_{n}^{\prime \prime}\right)+B_{n}\left(B_{n}^{\prime \prime}+\beta_{n}^{\prime \prime}\right)+C_{n}\left(C_{n}^{\prime \prime}+\gamma_{n}^{\prime \prime}\right)\right]_{r=a} \\
& =-\sum_{m=1}^{2} W_{m}\left[H_{1 m}(\mu) \mu-H_{2 m}(\mu)\right] \sin \phi \cos \phi, \\
& \sum_{n=1}^{\infty}\left[A_{n}\left(A_{n}^{\prime \prime \prime}+\alpha_{n}^{\prime \prime \prime}\right)+B_{n}\left(B_{n}^{\prime \prime \prime}+\beta_{n}^{\prime \prime \prime}\right)+C_{n}\left(C_{n}^{\prime \prime \prime}+\gamma_{n}^{\prime \prime \prime}\right)\right]_{r=a} \\
& =-a \Omega\left(1-\mu^{2}\right)^{1 / 2} \cos \phi \\
& +\sum_{m=1}^{2} W_{m} H_{1 m}(\mu)\left(1-\mu^{2}\right)^{1 / 2} \cos \phi,
\end{aligned}
$$

where

$$
\begin{aligned}
& H_{1 m}(\mu)= k T\left[1-(-1)^{m} \alpha\right] \frac{\left|\nabla n^{\infty}\right|}{n^{\infty}(\mathbf{0})} \mu \\
&+\frac{1}{a} \sum_{n=1}^{\infty} R_{m n} \delta_{n}^{(3)}(a, \mu), \\
& H_{2 m}(\mu)=k T\left[1-(-1)^{m} \alpha\right] \frac{\left|\nabla n^{\infty}\right|}{n^{\infty}(\mathbf{0})} \\
&+\frac{1}{a\left(1-\mu^{2}\right)^{1 / 2}} \sum_{n=1}^{\infty} R_{m n} \delta_{n}^{(1)}(a, \mu),
\end{aligned}
$$

and the function $\delta_{n}^{(3)}(r, \mu)$ is defined by Eq. (B.3). The first $2 M$ coefficients $R_{m n}$ have been determined through the procedure given in the previous subsection.

Careful examination of Eqs. (30a)-(30c) shows that the solution of the coefficient matrix generated is independent of the $\phi$ coordinate of the boundary points on the surface of the sphere $r=a$. Thus, these relations can be satisfied by utilizing the collocation technique presented for the solution of the electrochemical potential field. At the particle surface, Eqs. (30a)-(30c) are applied at $N$ discrete points (values of $\theta$ between 0 and $\pi$ ) and the infinite series in Eq. (29) are truncated after $N$ terms. This generates a set of $3 N$ linear algebraic equations for the $3 N$ unknown coefficients $A_{n}, B_{n}$, and $C_{n}$. The fluid velocity field is completely obtained once these coefficients are solved for a sufficiently large value of $N$.

\subsection{Derivation of the particle velocities}

The drag force and torque exerted by the fluid on the spherical particle can be determined from [32]

$\mathbf{F}=-8 \pi \eta A_{1} \mathbf{e}_{x}$,
$\mathbf{T}=-8 \pi \eta C_{1} \mathbf{e}_{y}$.

These expressions show that only the lowest-order coefficients $A_{1}$ and $C_{1}$ in Eq. (29) contribute to the hydrodynamic force and couple acting on the particle.

Because the particle is freely suspended in the surrounding fluid, the net force and torque exerted on the particle must vanish. Applying this constraint to Eq. (32), one has

$A_{1}=C_{1}=0$.

To determine the translational and angular velocities $U$ and $\Omega$ of the particle, Eq. (33) and the $3 N$ algebraic equations resulting from Eq. (30) are to be solved simultaneously.

\section{Results and discussion for diffusiophoresis}

The solution for the diffusiophoretic motion of a charged spherical particle parallel to two plane walls at an arbitrary position between them, obtained using the boundary collocation method described in the previous section, is presented in this section. The system of linear algebraic equations to be solved for the coefficients $R_{1 n}$ and $R_{2 n}$ is constructed from Eq. (20), while that for $A_{n}, B_{n}$, and $C_{n}$ is derived from Eq. (30). All the numerical integrations to evaluate the primed $\alpha_{n}, \beta_{n}$, and $\gamma_{n}$ as well as $\delta_{n}^{(i)}$ functions were done by 80-point Gauss-Laguerre quadrature.

When the points along the semicircular generating arc of the sphere (with a constant value of $\phi$ ) where the boundary conditions are to be exactly satisfied are being specified, the first points that should be chosen are $\theta=0$ and $\pi$, since these points define the projected area of the particle normal to the direction of motion and control the gaps between the particle and the neighboring plates. In addition, the point $\theta=\pi / 2$ is also important. However, an examination of the systems of linear algebraic equations (20) and (30) shows that the matrix equations become singular if these points are used. To overcome this difficulty, these points are replaced by closely adjacent points, i.e., $\theta=\delta, \pi / 2-\delta, \pi / 2+\delta$, and $\pi-\delta$ $[30,32]$. Additional points along the boundary are selected as mirror-image pairs about the plane $\theta=\pi / 2$ to divide the two quarter-circular arcs of the particle into equal segments. The optimum value of $\delta$ in this work is found to be $0.1^{\circ}$, for which the numerical results of the particle velocities converge satisfactorily. In selecting the boundary points, any value of $\phi$ may be used except for $\phi=0, \pi / 2$, and $\pi$ since the matrix equation (30) is singular for these values.

\subsection{Motion parallel to a single plane wall}

The boundary effects on diffusiophoresis are complicated functions of the properties of the particle and suspending solution, the electrochemical condition of the boundary, and the particle-boundary separation distance. Some typical collocation solutions for the translational and rotational velocities of a dielectric sphere undergoing diffusiophoresis 
Table 1

Normalized translational and rotational velocities of a spherical particle undergoing diffusiophoresis parallel to a single impermeable and nonconducting plane wall computed from the exact boundary-collocation solution and the asymptotic method-of-reflection solution for the case $Z=1, f_{1}=0.2$, $\kappa a=100$, and $\zeta e / k T=2$

\begin{tabular}{|c|c|c|c|c|}
\hline \multirow[t]{2}{*}{$a / b$} & \multicolumn{2}{|l|}{$U / U_{0}$} & \multicolumn{2}{|c|}{$-a \Omega / U_{0}$} \\
\hline & $\begin{array}{l}\text { Exact } \\
\text { solution }\end{array}$ & $\begin{array}{l}\text { Asymptotic } \\
\text { solution }\end{array}$ & $\begin{array}{l}\text { Exact } \\
\text { solution }\end{array}$ & $\begin{array}{l}\text { Asymptotic } \\
\text { solution }\end{array}$ \\
\hline \multicolumn{5}{|c|}{$\alpha=0$} \\
\hline 0.1 & 0.99992 & 0.99992 & 0.00002 & 0.00002 \\
\hline 0.2 & 0.99934 & 0.99935 & 0.00030 & 0.00030 \\
\hline 0.3 & 0.99788 & 0.99798 & 0.00154 & 0.00152 \\
\hline 0.4 & 0.99521 & 0.99578 & 0.00494 & 0.00480 \\
\hline 0.5 & 0.99103 & 0.99316 & 0.01242 & 0.01172 \\
\hline 0.6 & 0.98490 & 0.99115 & 0.02702 & 0.02430 \\
\hline 0.7 & 0.97580 & 0.99152 & 0.05412 & 0.04502 \\
\hline 0.8 & 0.96060 & 0.99694 & 0.10518 & 0.07680 \\
\hline 0.9 & 0.92322 & 1.01113 & 0.21643 & 0.12302 \\
\hline 0.95 & 0.85475 & & 0.34835 & \\
\hline 0.99 & 0.2493 & & 0.7542 & \\
\hline \multicolumn{5}{|c|}{$\alpha=-0.2$} \\
\hline 0.1 & 0.99969 & 0.99969 & 0.00002 & 0.00002 \\
\hline 0.2 & 0.99755 & 0.99758 & 0.00030 & 0.00030 \\
\hline 0.3 & 0.99169 & 0.99200 & 0.00156 & 0.00152 \\
\hline 0.4 & 0.97982 & 0.98159 & 0.00510 & 0.00480 \\
\hline 0.5 & 0.95838 & 0.96544 & 0.01323 & 0.01172 \\
\hline 0.6 & 0.92042 & 0.94326 & 0.03018 & 0.02430 \\
\hline 0.7 & 0.84954 & 0.91547 & 0.06473 & 0.04502 \\
\hline 0.8 & 0.69592 & 0.88342 & 0.13897 & 0.07680 \\
\hline 0.9 & 0.21212 & 0.84950 & 0.33712 & 0.12302 \\
\hline 0.95 & -0.75566 & & 0.63714 & \\
\hline 0.99 & -8.8227 & & 2.2435 & \\
\hline
\end{tabular}

parallel to a plane wall (with $c \rightarrow \infty$ ) for different values of the parameters $\alpha$ and $a / b$ are presented in Tables 1 and 2 for the cases of an impermeable and nonconducting wall and a wall with the imposed far-field electrolyte concentration gradient, respectively. The corresponding velocity for the diffusiophoretic motion of the particle in an infinite fluid, $U_{0}$, given by Eq. (7), is used to normalize the boundary-corrected values. All of the results obtained under the collocation scheme converge satisfactorily to at least the significant figures shown in the tables. The accuracy and convergence behavior of the truncation technique are principally a function of the ratio $a / b$. For the difficult case with $a / b=0.99$, the numbers of collocation points $M=40$ and $N=40$ are sufficiently large to achieve this convergence. For some cases, the particle will reverse the direction of diffusiophoresis and the magnitude of its normalized velocity can be dramatically varied when the separation distance is increased. Note that the situations associated with $\alpha=0$ and $\alpha=-0.2$ (taking $Z=1$ and $f_{1}=0.2$ ) in the tables are close to the particle migration in the aqueous solutions of $\mathrm{KCl}$ and $\mathrm{NaCl}$, respectively.

Through the use of spherical bipolar coordinates, Keh and Chen [24] obtained numerical solutions for the normalized translational and rotational velocities of a dielectric sphere surrounded by an infinitesimally thin electrical dou-
Table 2

Normalized translational and rotational velocities of a spherical particle undergoing diffusiophoresis parallel to a single plane wall prescribed with the far-field electrolyte concentration profile computed from the exact boundary-collocation solution and the asymptotic method-of-reflection solution for the case $Z=1, f_{1}=0.2, \kappa a=100$, and $\zeta e / k T=2$

\begin{tabular}{|c|c|c|c|c|}
\hline \multirow[t]{2}{*}{$a / b$} & \multicolumn{2}{|l|}{$U / U_{0}$} & \multicolumn{2}{|c|}{$-a \Omega / U_{0}$} \\
\hline & $\begin{array}{l}\text { Exact } \\
\text { solution }\end{array}$ & $\begin{array}{l}\text { Asymptotic } \\
\text { solution }\end{array}$ & $\begin{array}{l}\text { Exact } \\
\text { solution }\end{array}$ & $\begin{array}{l}\text { Asymptotic } \\
\text { solution }\end{array}$ \\
\hline \multicolumn{5}{|c|}{$\alpha=0$} \\
\hline 0.1 & 0.99984 & 0.99984 & 0.00002 & 0.00002 \\
\hline 0.2 & 0.99872 & 0.99873 & 0.00030 & 0.00030 \\
\hline 0.3 & 0.99573 & 0.99588 & 0.00154 & 0.00152 \\
\hline 0.4 & 0.99000 & 0.99078 & 0.00496 & 0.00480 \\
\hline 0.5 & 0.98046 & 0.98340 & 0.01252 & 0.01172 \\
\hline 0.6 & 0.96550 & 0.97430 & 0.02736 & 0.02430 \\
\hline 0.7 & 0.94215 & 0.96475 & 0.05498 & 0.04502 \\
\hline 0.8 & 0.90370 & 0.95699 & 0.10651 & 0.07680 \\
\hline 0.9 & 0.82932 & 0.95425 & 0.21260 & 0.12302 \\
\hline 0.95 & 0.75204 & & 0.32114 & \\
\hline 0.99 & 0.5976 & & 0.5205 & \\
\hline \multicolumn{5}{|c|}{$\alpha=-0.2$} \\
\hline 0.1 & 1.00006 & 1.00006 & 0.00002 & 0.00002 \\
\hline 0.2 & 1.00050 & 1.00050 & 0.00030 & 0.00030 \\
\hline 0.3 & 1.00188 & 1.00186 & 0.00152 & 0.00152 \\
\hline 0.4 & 1.00511 & 1.00497 & 0.00480 & 0.00480 \\
\hline 0.5 & 1.01179 & 1.01112 & 0.01174 & 0.01172 \\
\hline 0.6 & 1.02480 & 1.02218 & 0.02447 & 0.02430 \\
\hline 0.7 & 1.04930 & 1.04080 & 0.04620 & 0.04502 \\
\hline 0.8 & 1.09390 & 1.07050 & 0.08474 & 0.07680 \\
\hline 0.9 & 1.16276 & 1.11587 & 0.18095 & 0.12302 \\
\hline 0.95 & 1.17697 & & 0.33196 & \\
\hline 0.99 & 1.0353 & & 0.7646 & \\
\hline
\end{tabular}

ble layer undergoing electrophoresis parallel to a nonconducting plane wall. These solutions, which can apply to the case of diffusiophoresis of a sphere in the limit of Eq. (6) parallel to an impermeable plane wall, can be used to check the correctness of our collocation solutions. It has been found that our solutions for the particle velocities in this limiting case agree excellently with the bipolar-coordinate solutions, although the comparison is not shown here for conciseness.

In Appendix A, an approximate analytical solution for the same diffusiophoretic motion as that considered here is also obtained using a method of reflections. The translational and angular velocities of a spherical particle near a lateral plate are given by Eqs. (A.14a) and (A.14b), which are power series expansions in $\lambda(=a / b)$. The values of the wallcorrected normalized particle velocities calculated from this asymptotic solution, with the $O\left(\lambda^{6}\right)$ term neglected, are also listed in Tables 1 and 2 for comparison. It can be seen that the asymptotic formula of Eq. (A.14a) resulting from the method of reflections for $U / U_{0}$ agrees very well with the exact results as long as $\lambda \leqslant 0.5$; the errors in all cases are less than $0.8 \%$. However, the accuracy of Eqs. (A.14a) and (A.14b) [especially Eq. (A.14b) for $a \Omega / U_{0}$, in which the leading term is $O\left(\lambda^{4}\right)$ ] deteriorates rapidly, as expected, when the relative spacing between the particle and the plane wall becomes small. 


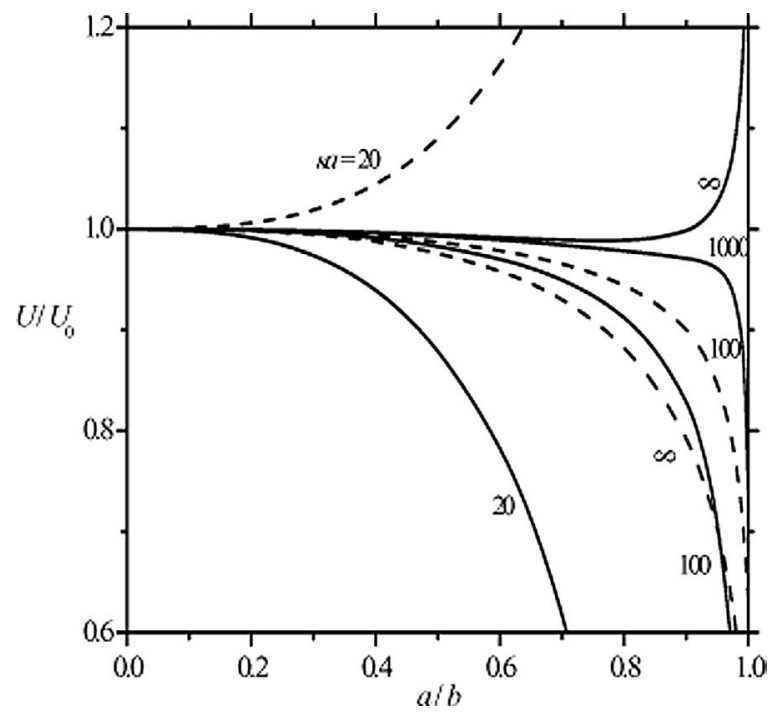

(a)

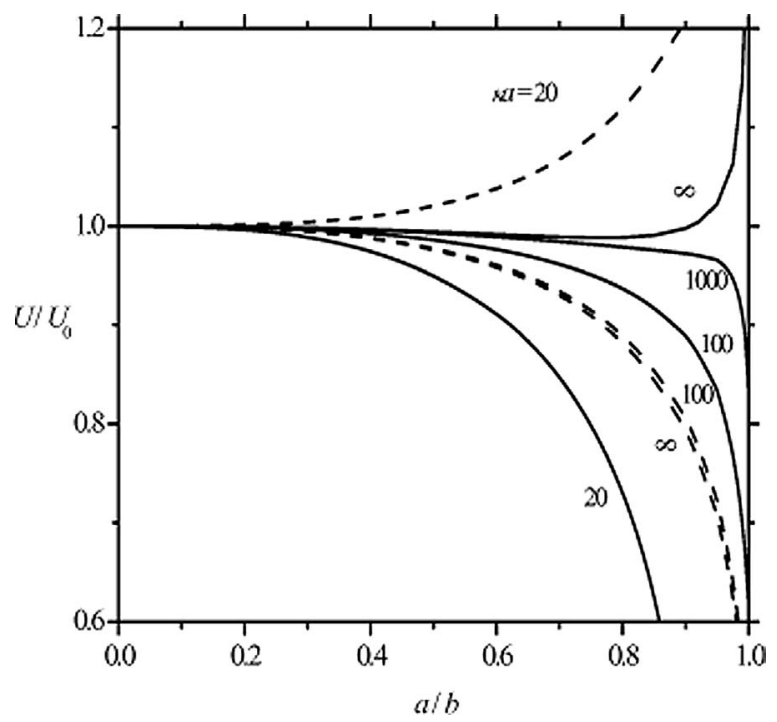

(b)

Fig. 2. Plots of the normalized diffusiophoretic mobility $U / U_{0}$ of a spherical particle parallel to a plane wall versus the separation parameter $a / b$ with $Z=1, f_{1}=0.2$, and $\zeta e / k T=-5$ for various values of $\kappa a$ : (a) $\alpha=0$; (b) $\alpha=-0.2$. The solid curves represent the case of an impermeable and nonconducting wall, and the dashed curves denote the case of a wall on which the far-field electrolyte concentration gradient is imposed.

Some exact numerical solutions for the normalized diffusiophoretic velocity $U / U_{0}$ of a spherical particle parallel to a plane wall as functions of $a / b$ are depicted in Fig. 2 for various values of $\kappa a$. It can be seen that, under the specified condition, $U / U_{0}$ increases with an increase in $\kappa a$ for the case of an impermeable and nonconducting wall [the boundary condition (12) is used], but decreases with an increase in $\kappa a$ for the case of a plane wall prescribed with the far-field electrolyte concentration distribution [the boundary condition (15) is used] for the otherwise specified condition. This increase and decrease in particle mobility become more pronounced as $a / b$ increases. This behavior is expected, knowing that the electrochemical potential gradients on the par- ticle surface near an impermeable and nonconducting wall increase as $\kappa a$ (or $G$ ) increases and these gradients near a wall with the imposed far-field concentration gradient decrease as $\kappa a$ (or $G$ ) increases for the present case (see the analysis in Appendix A).

Examination of the results in Fig. 2 reveals an interesting feature. For the case of a plane wall impermeable to the ions/charges under the situation of large $\kappa a$ (e.g., with $\kappa a \rightarrow \infty$ ), the diffusiophoretic mobility of the particle decreases with increased $a / b$ when $a / b$ is small, but increases from a minimum with increased $a / b$ when $a / b$ is sufficiently large. When the gap between the particle and the wall turns thin, the particle can even move faster than it would at $a / b=0$. For example, as $\kappa a \rightarrow \infty$ and when $a / b=0.99$, the diffusiophoretic velocity for a typical case can be as much as $15 \%$ higher than the value with the wall far away from the particle. Under the situation of relatively small $\kappa a$, the diffusiophoretic mobility of the particle near the impermeable and nonconducting wall is a monotonic decreasing function of $a / b$. For a case where a linear electrochemical potential profile is prescribed on the plane wall, which is consistent with the far-field distribution under the situation of small $\kappa a$ (e.g., with $\kappa a=20$ ), the diffusiophoretic mobility of the particle may increase monotonically with the increase of $a / b$ from $a / b=0$. Under the situation of relatively large $\kappa a$, the diffusiophoretic mobility of the particle near the wall prescribed with the far-field electrolyte concentration distribution becomes a monotonic decreasing function of $a / b$. This interesting feature that $U / U_{0}$ may not be a monotonic decreasing function of $a / b$ and can even be greater than unity is understandable because the wall effect of hydrodynamic resistance on the particle is in competition with the wall effect of electrochemical enhancement when a particle with large $\kappa a$ is undergoing diffusiophoretic motion parallel to an impermeable and nonconducting plate or when a particle with small $\kappa a$ is moving near a lateral plate with the imposed far-field electrolyte concentration gradient. A careful examination of the asymptotic formula for $U / U_{0}$ given by Eq. (A.14a) shows good agreement of the numerical outcome in Fig. 2 with the analytical solution.

The results in Tables 1 and 2 indicate that the dielectric sphere undergoing diffusiophoresis parallel to a plane wall rotates in the direction opposite to that for a sphere migrating in the same direction but under a body-force field (e.g., a gravitational field). The explanation for this behavior is analogous to the case of electrophoresis of a charged sphere with a thin double layer parallel to a nonconducting plate [24]. For an otherwise specified condition, the magnitude of the normalized rotational velocity of the diffusiophoretic sphere near a given plane wall is a monotonically increasing function of $a / b$.

\subsection{Motion parallel to two plane walls}

Some converged collocation solutions for the normalized velocity $U / U_{0}$ of a charged spherical particle undergoing 
Table 3

Normalized diffusiophoretic velocity of a spherical particle along the median plane between two parallel plane walls computed from the exact boundary-collocation solution and the asymptotic method-of-reflection solution for the case $Z=1, f_{1}=0.2, \kappa a=100$, and $\zeta e / k T=2$

\begin{tabular}{|c|c|c|c|c|}
\hline \multirow[t]{3}{*}{$a / b$} & \multicolumn{4}{|l|}{$U / U_{0}$} \\
\hline & \multicolumn{2}{|l|}{$\alpha=0$} & \multicolumn{2}{|l|}{$\alpha=-0.2$} \\
\hline & $\begin{array}{l}\text { Exact } \\
\text { solution }\end{array}$ & $\begin{array}{l}\text { Asymptotic } \\
\text { solution }\end{array}$ & $\begin{array}{l}\text { Exact } \\
\text { solution }\end{array}$ & $\begin{array}{l}\text { Asymptotic } \\
\text { solution }\end{array}$ \\
\hline \multicolumn{5}{|c|}{ For impermeable plane walls } \\
\hline 0.1 & 0.99968 & 0.99968 & 0.99915 & 0.99915 \\
\hline 0.2 & 0.99751 & 0.99751 & 0.99325 & 0.99325 \\
\hline 0.3 & 0.99202 & 0.99207 & 0.97760 & 0.97768 \\
\hline 0.4 & 0.98232 & 0.98272 & 0.94781 & 0.94860 \\
\hline 0.5 & 0.96820 & 0.97005 & 0.89890 & 0.90342 \\
\hline 0.6 & 0.94992 & 0.95629 & 0.82267 & 0.84166 \\
\hline 0.7 & 0.92794 & 0.94567 & 0.70053 & 0.76285 \\
\hline 0.8 & 0.90191 & 0.94489 & 0.47632 & 0.67198 \\
\hline 0.9 & 0.86257 & 0.96346 & -0.12763 & 0.57489 \\
\hline 0.95 & 0.80313 & & -1.24283 & \\
\hline 0.99 & 0.1946 & & -10.226 & \\
\hline \multicolumn{5}{|c|}{ For plane walls prescribed with the far-field concentration profile } \\
\hline 0.1 & 0.99952 & 0.99952 & 0.99991 & 0.99991 \\
\hline 0.2 & 0.99620 & 0.99620 & 0.99939 & 0.99940 \\
\hline 0.3 & 0.98762 & 0.98764 & 0.99829 & 0.99843 \\
\hline 0.4 & 0.97197 & 0.97221 & 0.99712 & 0.99780 \\
\hline 0.5 & 0.94811 & 0.94953 & 0.99731 & 0.99951 \\
\hline 0.6 & 0.91521 & 0.92083 & 1.00195 & 1.00719 \\
\hline 0.7 & 0.87210 & 0.88937 & 1.01684 & 1.02650 \\
\hline 0.8 & 0.81568 & 0.86085 & 1.05193 & 1.06553 \\
\hline 0.9 & 0.73429 & 0.84380 & 1.11612 & 1.13524 \\
\hline 0.95 & 0.66825 & & 1.13957 & \\
\hline 0.99 & 0.5615 & & 1.0560 & \\
\hline
\end{tabular}

diffusiophoresis on the median plane between two parallel plane walls (with $c=b$ and $\Omega=0$ ) for various values of the parameters $\alpha$ and $a / b$ are presented in Table 3 for the two cases of impermeable and nonconducting walls and walls prescribed with the far-field electrolyte concentration distribution. The corresponding method-of-reflection solutions, given by Eq. (A.21) in Appendix A as a power series expansion in $\lambda(=a / b)$ correct to $O\left(\lambda^{5}\right)$, are also listed in this table for comparison. Similarly to the case of migration of a spherical particle parallel to a single plane wall considered in the previous section, the approximate analytical formula of Eq. (A.21) agrees very well with the exact results as long as $\lambda \leqslant 0.5$, but can have significant errors when $\lambda \geqslant 0.6$. In general, Eq. (A.21) overestimates the diffusiophoretic velocity of the particle. A comparison between Table 3 for the case of a slit and Tables 1 and 2 for the case of a single parallel plane indicates that the assumption that the boundary effect for two walls can be obtained by simple addition of single-wall effects in general leads to a smaller correction to diffusiophoretic motion when $a / b$ is small but can give a greater correction as $a / b$ becomes large.

In Fig. 3, typical collocation results for the normalized diffusiophoretic mobility $U / U_{0}$ of a dielectric sphere migrating on the median plane between two parallel plane walls are plotted as functions of $a / b$ for several values of $\alpha$

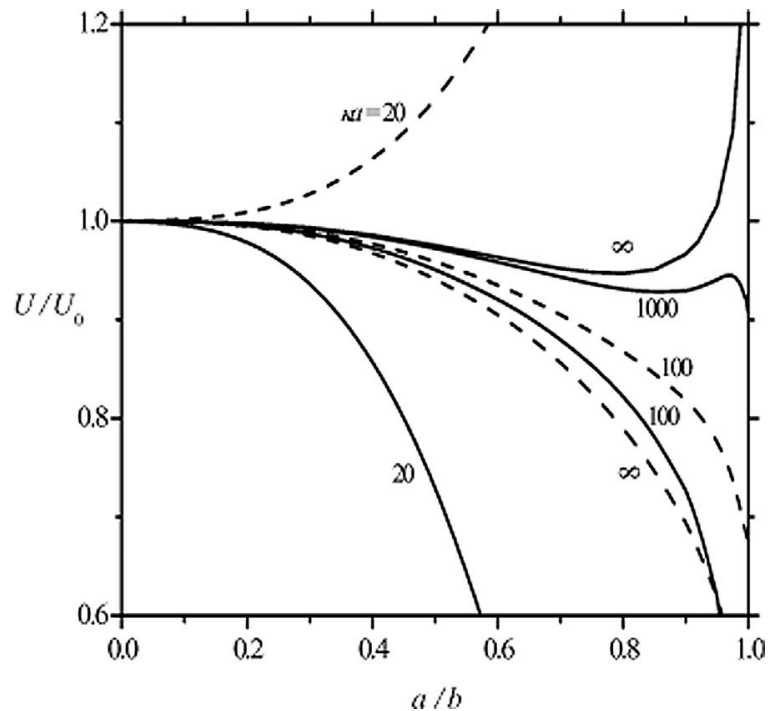

(a)

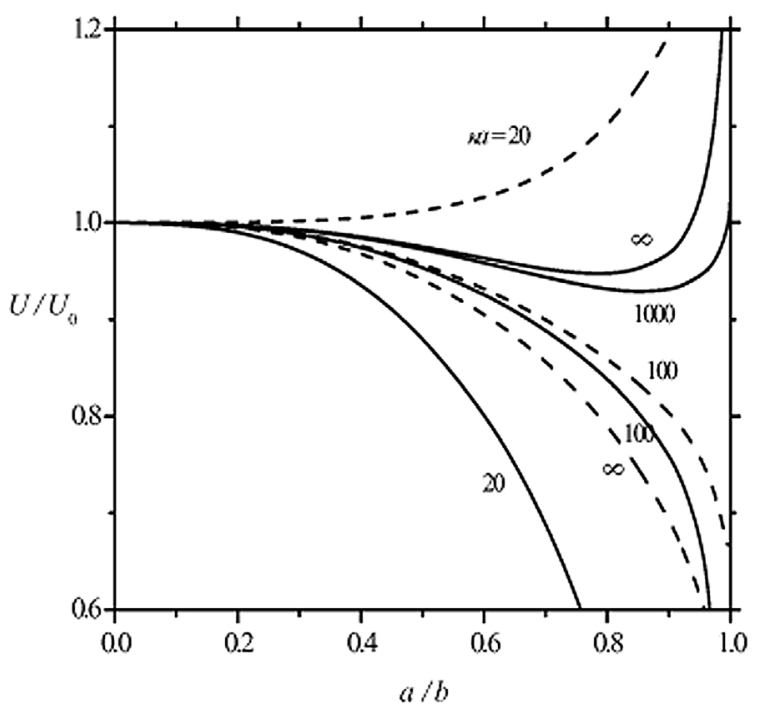

(b)

Fig. 3. Plots of the normalized diffusiophoretic mobility $U / U_{0}$ of a spherical particle migrating on the median plane between two parallel plane walls (with $c=b$ ) versus the separation parameter $a / b$ with $Z=1, f_{1}=0.2$, and $\zeta e / k T=-5$ for several values of $\kappa a$ : (a) $\alpha=0$; (b) $\alpha=-0.2$. The solid curves represent the case of impermeable and nonconducting walls, and the dashed curves denote the case of walls prescribed with the far-field electrolyte concentration distribution.

and $\kappa a$. Analogously to the corresponding motion of a particle parallel to a single plane wall under the same condition illustrated in Fig. 2, for a specified value of $a / b, U / U_{0}$ decreases with an increase in $\kappa a$ for the case of walls with the imposed far-field electrolyte concentration gradient and increases with an increase in $\kappa a$ for the case of impermeable and nonconducting walls. Again, for the case of impermeable and nonconducting walls in the situation of large $\kappa a$, the diffusiophoretic mobility of the particle may first go through a minimum with the increase of $a / b$ from $a / b=0$ and then increase monotonically, and the particle can even move faster than it would at $a / b=0$. Also, for the case 
of walls prescribed with the far-field electrolyte concentration distribution under the situation of small $\kappa a$, the particle mobility may increase monotonically with an increase in $a / b$ from $a / b=0$. This result indicates that the effect of electrochemical enhancement, rather than that of hydrodynamic resistance, can be overriding when the particle-wall gap thickness is small. An examination of the asymptotic formula for $U / U_{0}$ in Eq. (A.21) also shows good agreement of the trend in Fig. 3 with the analytical solution.

A careful comparison of the curves in Fig. 3 for the case of a slit with the corresponding curves in Fig. 2 for the case of a single wall reveals an interesting feature of the boundary effect on diffusiophoresis of a colloidal sphere. The presence of a second, identical, lateral plane wall, even at a symmetric position with respect to the sphere against the first, does not always enhance the wall effect on the diffusiophoretic particle induced by the first plate only. This result reflects again the fact that the lateral wall can affect the electrochemical driving force and the viscous drag force on a particle in opposite directions. Each force is increased in its own direction as the value of $a / b$ turns small, but to a different degree, for the case of diffusiophoretic motion of a particle in a slit relative to that for the case of migration parallel to a single plate. Thus, the net effect composed of these two opposite forces for the slit case is not necessarily to enhance that for the case of a single wall.

Fig. 4 shows the collocation results for the normalized translational velocity $U / U_{0}$ and rotational velocity $a \Omega / U_{0}$ of a charged sphere undergoing diffusiophoresis parallel to two impermeable and nonconducting plane walls at various positions between them for a typical case. The dashed curves (with $a / b=$ constant) illustrate the effect of the position of the second wall (at $z=c$ ) on the particle velocities for various values of the relative sphere-to-first-wall spacing $b / a$. The solid curves [with $2 a /(b+c)=$ constant] indicate the variation of the particle velocities as functions of the sphere position at various values of the relative wall-to-wall spacing $(b+c) / 2 a$. As illustrated in Fig. 4a, the net wall effect for the given case is to reduce the diffusiophoretic mobility $U / U_{0}$ of the particle. At a constant value of $2 a /(b+c)$, the particle in general (with exceptions) experiences a minimum viscous drag force and has a greatest translational velocity (without rotation) when it is located midway between the two walls (with $c=b$ ); the hydrodynamic drag increases, the translational velocity decreases, and the rotational velocity increases as the particle approaches either of the walls [or the ratio $b /(b+c)$ decreases]. At a specified value of $a / b$ for the diffusiophoretic particle near a first lateral wall, the presence of a second plate is to further reduce the translational and rotational velocities of the particle, and the degree of this reduction increases monotonically with a decrease in the relative distance between the particle and the second plate [or with an increase in $b /(b+c)$ ].

On the other hand, for some cases such as the diffusiophoresis of a dielectric sphere with a large value of $\kappa a$ parallel to two impermeable and nonconducting plane walls or

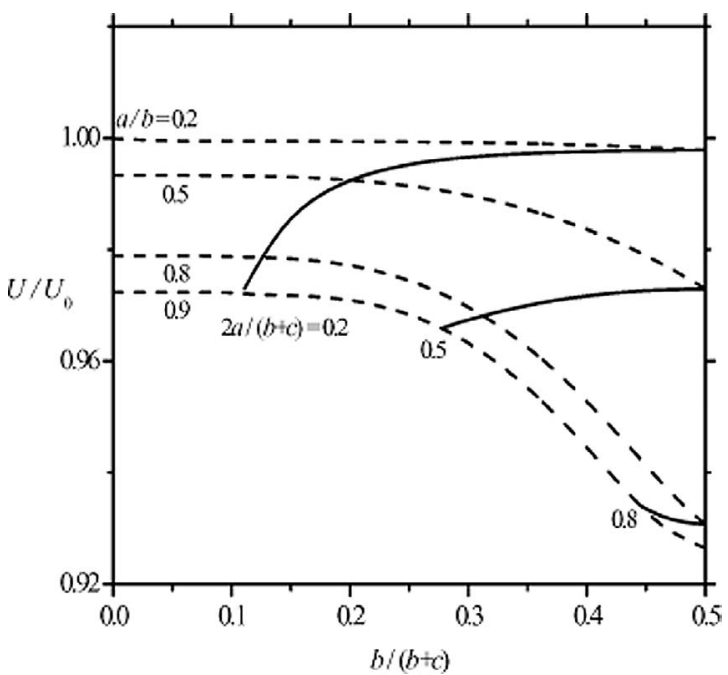

(a)

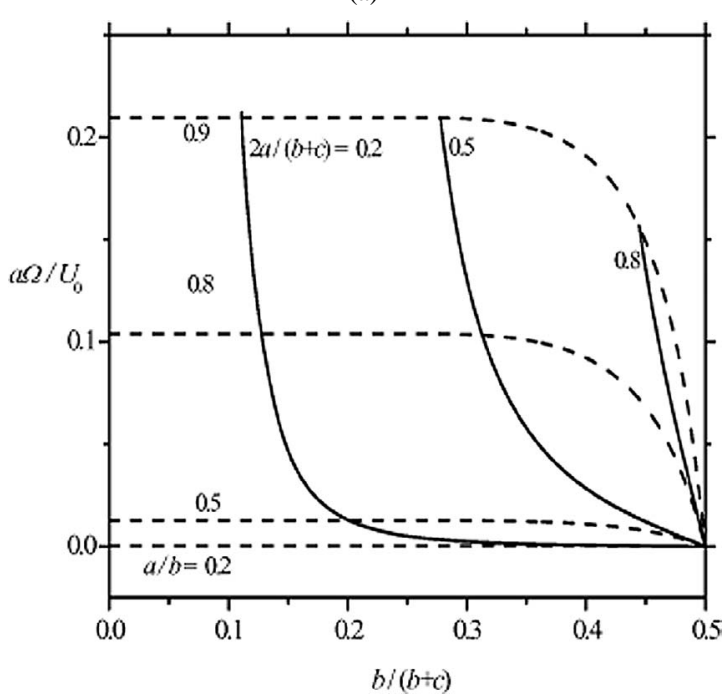

(b)

Fig. 4. Plots of the normalized velocities of a spherical particle undergoing diffusiophoresis parallel to two impermeable and nonconducting plane walls versus the ratio $b /(b+c)$ for the case of $Z=1, f_{1}=f_{2}=0.2$, $\kappa a=1000$, and $|\zeta e / k T|=5$ with $a / b$ and $2 a /(b+c)$ as parameters: (a) translational velocity $U / U_{0} ;$ (b) rotational velocity $a \Omega / U_{0}$.

with a small value of $\kappa a$ parallel to two plates prescribed with the far-field electrolyte concentration distribution, the net wall effect can increase the diffusiophoretic mobility of the particle relative to its isolated value. At a fixed value of $2 a /(b+c)$ in these cases, the normalized particle mobility has a relatively small value as it is located midway between the two walls, where the particle experiences a minimum effect of electrochemical enhancement, and becomes relatively large when it approaches either of the walls. At a given value of $a / b$ for the diffusiophoretic particle and the first lateral plate, the effect induced by the presence of the second plate on the particle mobility is not necessarily a monotonic function of its distance from the particle. This dependence is quite complicated and is not graphically presented here, for conciseness. 


\section{Electrophoresis}

In this section, we consider the steady electrophoretic motion of a dielectric sphere in a uniformly applied electric field $\mathbf{E}^{\infty}=E^{\infty} \mathbf{e}_{x}$ parallel to two plane walls. The translational and angular velocities of the particle caused by the field are $\mathbf{U}=U \mathbf{e}_{x}$ and $\boldsymbol{\Omega}=\Omega \mathbf{e}_{y}$, respectively. The bulk concentration $n^{\infty}$ of the symmetric electrolyte in the fluid beyond the electric double layer is constant now. Like the analysis in Section 2, the thickness of the double layer is assumed to be much smaller than the radius of the particle and the surface-to-surface distance between the particle and each wall, but the polarization effect in the thin diffuse layer is incorporated.

Outside the double layer, the electrochemical potentials $\mu_{m}$ of the ions still satisfy Laplace's equation (8) and boundary conditions (10)-(15), but their undisturbed values in Eq. (14) are replaced by

$\mu_{m \infty}=\mu_{m}^{0}+k T \ln n^{\infty}-(-1)^{m} Z e E^{\infty} x$.

The solution for $\mu_{m}$ in this case can still be expressed as Eq. (19) with coefficients $R_{m n}$ determined by

$$
\begin{aligned}
& \sum_{n=1}^{\infty} \sum_{m=1}^{2} R_{m n} R_{m}^{\prime}(\mu) \\
& \quad=Z e E^{\infty}\left(a-2 \beta_{11}+2 \beta_{12}\right)\left(1-\mu^{2}\right)^{1 / 2}
\end{aligned}
$$

$$
\begin{aligned}
& \sum_{n=1}^{\infty} \sum_{m=1}^{2} R_{m n} R_{m}^{\prime \prime}(\mu) \\
& \quad=-Z e E^{\infty}\left(a-2 \beta_{22}+2 \beta_{21}\right)\left(1-\mu^{2}\right)^{1 / 2},
\end{aligned}
$$

to replace Eq. (20). The governing equations, boundary conditions, and solution for the fluid velocity field have the same forms as those given by Eqs. (22)-(29). The final results for the translational and angular velocities of the particle can be determined by the simultaneous solution of Eqs. (30) and (33), with

$$
H_{1 m}(\mu)=-(-1)^{m} Z e E^{\infty} \mu+\frac{1}{a} \sum_{n=1}^{\infty} R_{m n} \delta_{n}^{(3)}(a, \mu),
$$

$H_{2 m}(\mu)=-(-1)^{m} Z e E^{\infty}$

$$
+\frac{1}{a\left(1-\mu^{2}\right)^{1 / 2}} \sum_{n=1}^{\infty} R_{m n} \delta_{n}^{(1)}(a, \mu),
$$

in replacement for Eq. (31).

Some converged collocation solutions for the translational and rotational velocities of a dielectric sphere undergoing electrophoresis parallel to a plane wall (with $c \rightarrow \infty$ ) for various values of $a / b$ are presented in Table 4 , while the corresponding results for the electrophoretic velocity of the particle on the median plane between two parallel plates (with $c=b$ ) are given in Table 5. Now, the electrophoretic velocity of the particle in an unbounded fluid
Table 4

\begin{tabular}{|c|c|c|c|c|}
\hline \multirow[t]{2}{*}{$a / b$} & \multicolumn{2}{|l|}{$U / U_{0}$} & \multicolumn{2}{|c|}{$-a \Omega / U_{0}$} \\
\hline & $\begin{array}{l}\text { Exact } \\
\text { solution }\end{array}$ & $\begin{array}{l}\text { Asymptotic } \\
\text { solution }\end{array}$ & $\begin{array}{l}\text { Exact } \\
\text { solution }\end{array}$ & $\begin{array}{l}\text { Asymptotic } \\
\text { solution }\end{array}$ \\
\hline \multicolumn{5}{|c|}{ For a nonconducting plane wall } \\
\hline 0.1 & 0.99994 & 0.99994 & 0.00002 & 0.00002 \\
\hline 0.2 & 0.99950 & 0.99952 & 0.00030 & 0.00030 \\
\hline 0.3 & 0.99842 & 0.99853 & 0.00154 & 0.00152 \\
\hline 0.4 & 0.99655 & 0.99709 & 0.00493 & 0.00480 \\
\hline 0.5 & 0.99389 & 0.99571 & 0.01235 & 0.01172 \\
\hline 0.6 & 0.99054 & 0.99556 & 0.02675 & 0.02430 \\
\hline 0.7 & 0.98685 & 0.99853 & 0.05320 & 0.04502 \\
\hline 0.8 & 0.98377 & 1.00740 & 0.10222 & 0.07680 \\
\hline 0.9 & 0.98557 & 1.02603 & 0.20582 & 0.12302 \\
\hline 0.95 & 0.99640 & & 0.32277 & \\
\hline 0.99 & 1.0898 & & 0.6057 & \\
\hline \multicolumn{5}{|c|}{ For a plane wall prescribed with the far-field potential profile } \\
\hline 0.1 & 0.99982 & 0.99982 & 0.00002 & 0.00002 \\
\hline 0.2 & 0.99856 & 0.99856 & 0.00030 & 0.00030 \\
\hline 0.3 & 0.99520 & 0.99532 & 0.00154 & 0.00152 \\
\hline 0.4 & 0.98868 & 0.98948 & 0.00498 & 0.00480 \\
\hline 0.5 & 0.97772 & 0.98085 & 0.01259 & 0.01172 \\
\hline 0.6 & 0.96031 & 0.96988 & 0.02762 & 0.02430 \\
\hline 0.7 & 0.93278 & 0.95774 & 0.05574 & 0.04502 \\
\hline 0.8 & 0.88706 & 0.94652 & 0.10842 & 0.07680 \\
\hline 0.9 & 0.80016 & 0.93935 & 0.21538 & 0.12302 \\
\hline 0.95 & 0.71488 & & 0.32020 & \\
\hline 0.99 & 0.5591 & & 0.4984 & \\
\hline
\end{tabular}

Normalized translational and rotational velocities of a spherical particle undergoing electrophoresis parallel to a single plane wall computed from the exact boundary-collocation solution and the asymptotic method-ofreflection solution for the case $Z=1, f_{1}=f_{2}=0.2, \kappa a=100$, and $\zeta e / k T=2$

Table 5

Normalized electrophoretic velocity of a spherical particle along the median plane between two plane walls computed from the exact boundarycollocation solution and the asymptotic method-of-reflection solution for the case $Z=1, f_{1}=f_{2}=0.2, \kappa a=100$, and $\zeta e / k T=2$

\begin{tabular}{llllll}
\hline$a / b$ & \multicolumn{2}{l}{$U / U_{0}$} & & & \\
& \multicolumn{2}{l}{$\begin{array}{l}\text { For nonconducting } \\
\text { plane walls }\end{array}$} & & \multicolumn{2}{l}{$\begin{array}{l}\text { For plane walls prescribed with } \\
\text { the far-field potential profile }\end{array}$} \\
\cline { 2 - 3 } & $\begin{array}{l}\text { Exact } \\
\text { solution }\end{array}$ & $\begin{array}{l}\text { Asymptotic } \\
\text { solution }\end{array}$ & & $\begin{array}{l}\text { Exact } \\
\text { solution }\end{array}$ & $\begin{array}{l}\text { Asymptotic } \\
\text { solution }\end{array}$ \\
\hline 0.1 & 0.99973 & 0.99973 & & 0.99948 & 0.99948 \\
& 0.99788 & 0.99791 & & 0.99592 & 0.99591 \\
0.2 & 0.99328 & 0.99340 & & 0.98669 & 0.98664 \\
0.3 & 0.98534 & 0.98586 & & 0.96977 & 0.96985 \\
0.4 & 0.97426 & 0.97619 & & 0.94381 & 0.94493 \\
0.5 & 0.96105 & 0.96690 & & 0.90762 & 0.91288 \\
0.6 & 0.94784 & 0.96253 & & 0.85944 & 0.87674 \\
0.7 & 0.93916 & 0.97005 & 0.79502 & 0.84199 \\
0.8 & 0.94937 & 0.99928 & 0.70090 & 0.81694 \\
0.9 & 0.98303 & & 0.62704 & \\
0.95 & 1.1572 & & 0.5167 & \\
0.99 & & & &
\end{tabular}

given by Eq. (5) is used to normalize the wall-corrected values. The corresponding method-of-reflection solutions, given by Eqs. (A.14a), (A.14b), and (A.21) with the parameter $G$ defined by Eq. (A.16) in Appendix A as power series expansions in $\lambda(=a / b)$ correct to $O\left(\lambda^{5}\right)$, are also listed 


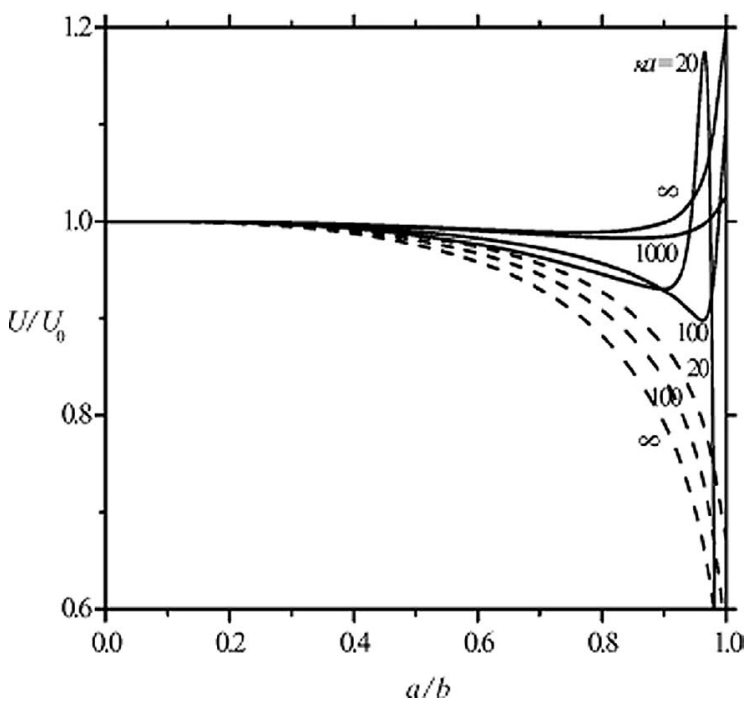

(a)

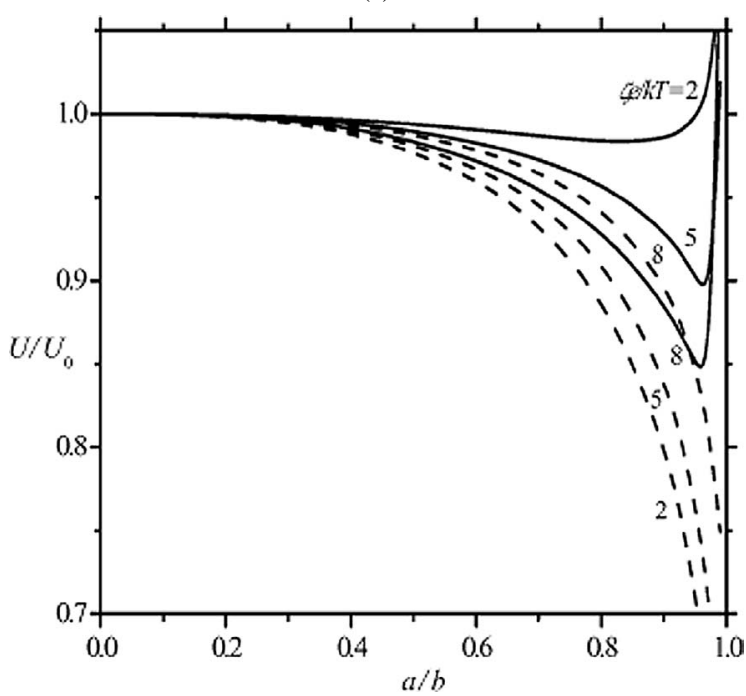

(b)

Fig. 5. Plots of the normalized electrophoretic velocity of a dielectric sphere parallel to a plane wall versus the separation parameter $a / b$ with $Z=1$ and $f_{1}=f_{2}=0.2$ : (a) $|\zeta e / k T|=5$; (b) $\kappa a=100$. The solid curves represent the case of an impermeable and nonconducting wall, and the dashed curves denote the case of a wall on which the far-field electrolyte concentration gradient is imposed.

in these tables for comparison. Analogously to the cases of diffusiophoresis considered in the previous section, the asymptotic formulas of Eqs. (A.14a) and (A.21) for $U / U_{0}$ agree quite well with the exact results as long as $\lambda \leqslant 0.6$, but can have significant errors for greater values of $\lambda$.

In Fig. 5, typical collocation results for the normalized electrophoretic mobility $U / U_{0}$ of a spherical particle parallel a plane wall as functions of $a / b$ are plotted for various values of $\kappa a$ and $\zeta e / k T$. The corresponding results for the particle undergoing electrophoresis on the median plane between two parallel plane walls are depicted in Fig. 6. For the case of nonconducting plane walls under the specified condition, the value of $U / U_{0}$ decreases with an increase in $a / b$ as $a / b$ is small, but increases from a minimum (to a max-

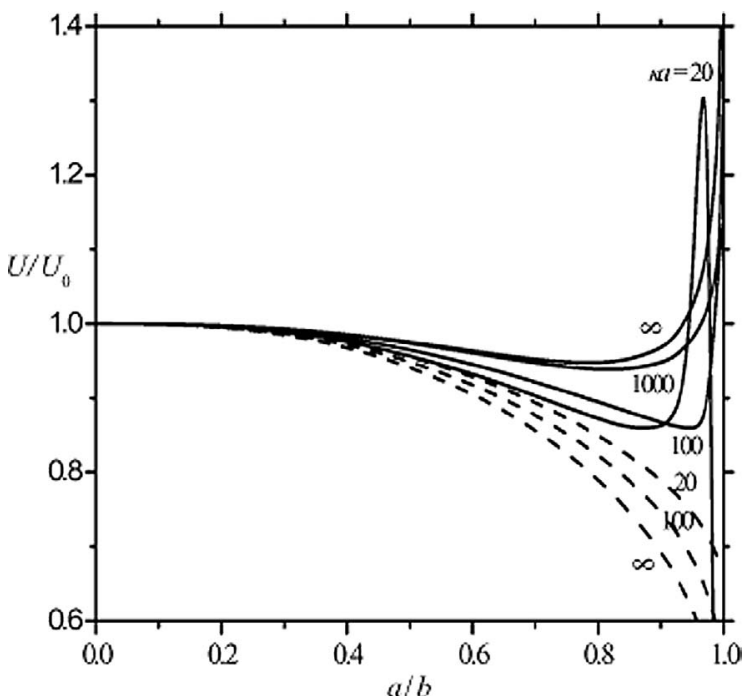

(a)

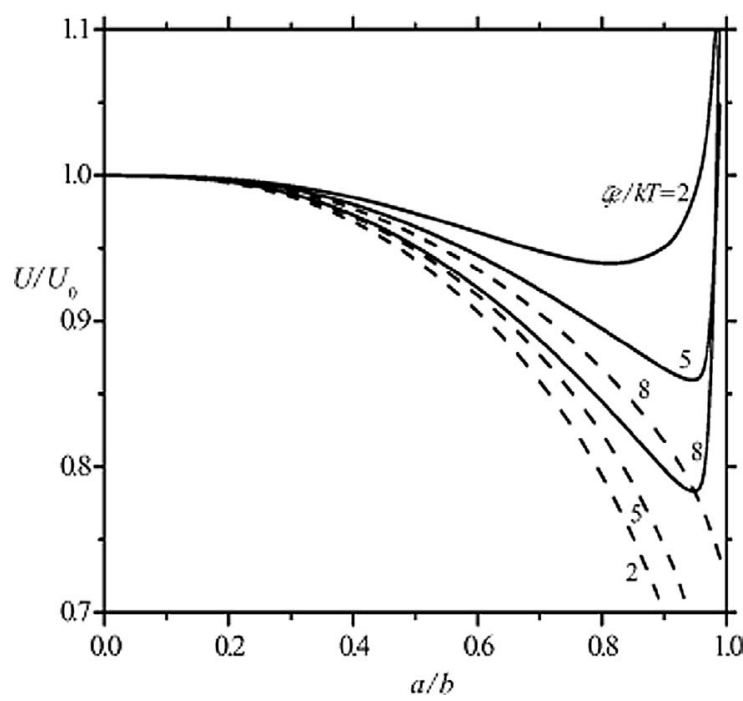

(b)

Fig. 6. Plots of the normalized electrophoretic velocity of a dielectric sphere migrating on the medium plane between two parallel plane walls versus the separation parameter $a / b$ with $Z=1$ and $f_{1}=f_{2}=0.2$ : (a) $|\zeta e / k T|=5$; (b) $\kappa a=100$. The solid curves represent the case of an impermeable and nonconducting wall, and the dashed curves denote the case of a wall on which the far-field electrolyte concentration gradient is imposed.

imum if the value of $\kappa a$ is small) with increasing $a / b$ and can be greater than unity as $a / b$ is sufficiently large. For the case of plane walls prescribed with the undisturbed electric potential distribution, $U / U_{0}$ is a monotonic decreasing function of $a / b$. It appears that $U / U_{0}$ in general increases with an increase in $\kappa a$ and with a decrease in $\zeta e / k T$ for the case of nonconducting walls, but decreases with an increase in $\kappa a$ and with a decrease in $\zeta e / k T$ for the case of plane walls prescribed with the undisturbed potential distribution. However, similar to the case of diffusiophoresis considered in the previous section, no simple rule could appropriately describe the boundary effects on the electrophoretic mobility of the particle, which are dependent on the combination of $\zeta e / k T, \kappa a, Z, f_{1}, f_{2}$, and $a / b$. 


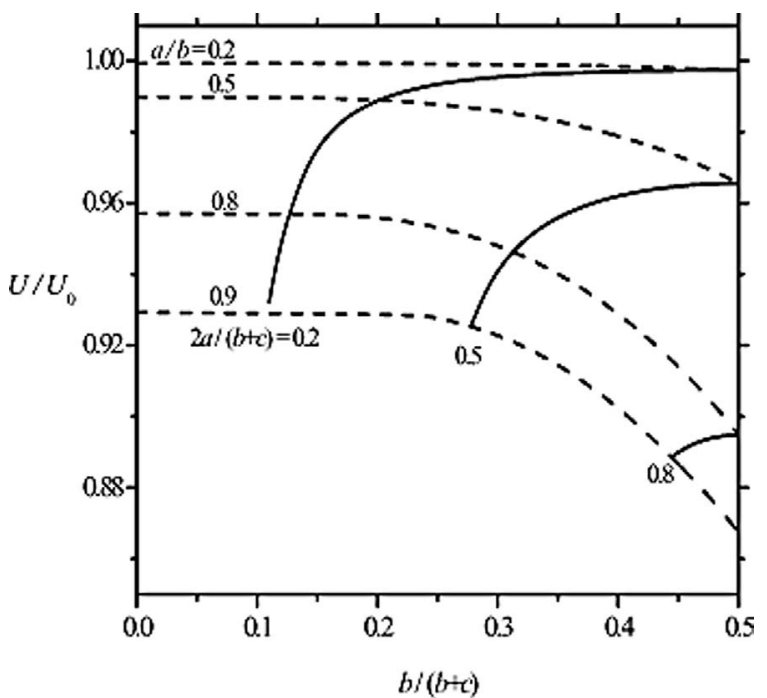

(a)

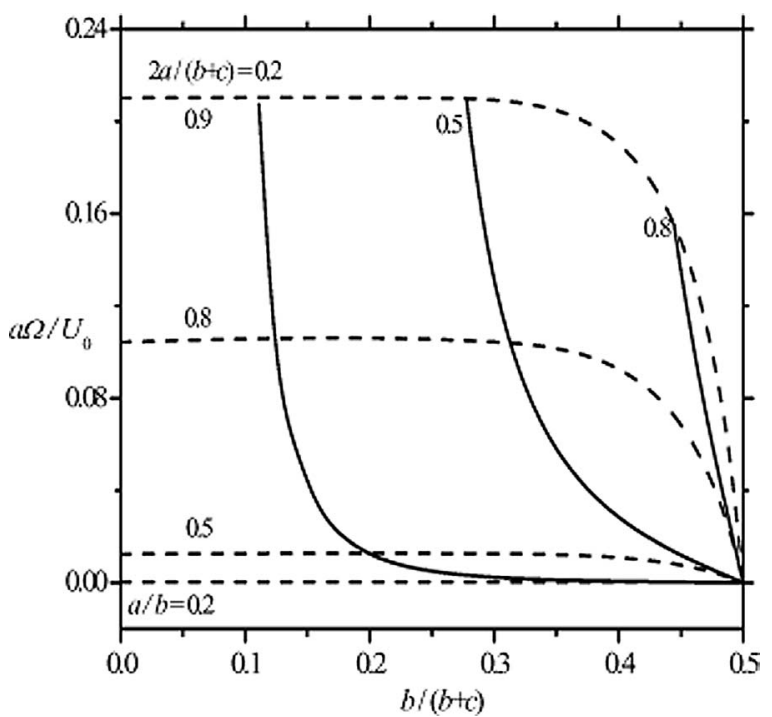

(b)

Fig. 7. Plots of the normalized velocities of a dielectric sphere undergoing electrophoresis parallel to two impermeable and nonconducting plane walls versus the ratio $b /(b+c)$ for the case of $Z=1, f_{1}=f_{2}=0.2, \kappa a=1000$, and $|\zeta e / k T|=5$ with $a / b$ and $2 a /(b+c)$ as parameters: (a) translational velocity $U / U_{0}$; (b) rotational velocity $a \Omega / U_{0}$.

Some collocation results for the normalized translational velocity $U / U_{0}$ and rotational velocity $a \Omega / U_{0}$ of a dielectric sphere undergoing electrophoresis parallel to two nonconducting plane walls at various positions between them are displayed in Fig. 7. Similar to the cases of diffusiophoresis, the particle experiences a minimum viscous drag and has a greatest translational velocity (without rotation) when it is located midway between the two walls (with $c=b$ ). At a specific value of $a / b$ for the electrophoretic particle near a first lateral wall, the presence of a second plate is to further reduce the translational and rotational velocities of the particle, and the degree of this reduction increases monotonically with a decrease in the relative distance between the particle and the second plate [or with an increase in $b /(b+c)$ ].

\section{Conclusions}

In this work, the exact numerical solutions and approximate analytical solutions for the quasi-steady diffusiophoretic and electrophoretic motions of a charged sphere parallel to two infinite plane walls at an arbitrary position between them have been obtained by using the boundarycollocation technique and the method of reflections, respectively. Both the cases of impermeable and nonconducting walls and of walls with the imposed far-field electrolyte concentration gradient were examined in the limit of vanishingly small Reynolds and Peclet numbers. It has been found that the boundary effects on these phoretic motions of a particle are quite significant and are complicated functions of the properties of the particle and surrounding ions ( $\zeta e / k T, \kappa a, Z, f_{1}$, and $f_{2}$ ), the electrochemical conditions of the boundaries, and the separation distances $(a / b$ and $a / c)$. The diffusiophoretic or electrophoretic mobility of a particle near a wall is generally, but not necessarily, a monotonic decreasing function of the separation parameter $a / b$. When the value of $a / b$ is sufficiently large, the effect of a lateral wall can speed up or slow down the particle velocity relative to its isolated value depending on the values of the relevant parameters of the particle-electrolyte system and the electrochemical boundary condition at the wall. This behavior reflects the competition between the relatively weak hydrodynamic retardation exerted by the neighboring wall on the particle migration and the possible, strong phoretic enhancement due to the electrochemical interaction between the particle and the lateral wall. No general rule can make an adequate prediction for such complicated phenomena present in the boundary effects on diffusiophoresis and electrophoresis.

\section{Acknowledgment}

This research was supported by the National Science Council of the Republic of China.

\section{Appendix A. Analysis of the diffusiophoresis and electrophoresis of a spherical particle parallel to one or two plane walls by a method of reflections}

In this Appendix, we analyze the quasi-steady diffusiophoretic and electrophoretic motions of a dielectric sphere of radius $a$ with uniform zeta potential $\zeta$ in the solution of a symmetric electrolyte either parallel to an infinite flat wall (with $c \rightarrow \infty$ ) or on the median plane between two parallel plates (with $c=b$ ), as shown in Fig. 1, by a method of reflections. The effect of the walls on the translational velocity $\mathbf{U}$ and angular velocity $\boldsymbol{\Omega}$ of the particle is sought in expansions of $\lambda$, which equals $a / b$, the ratio of the particle radius to the distance between the wall and the center of the particle. 


\section{A.1. Motion parallel to a plane wall}

For the problem of diffusiophoretic motion of a spherical particle driven by a uniform electrolyte concentration gradient $\nabla n^{\infty}$ parallel to an impermeable and nonconducting plane wall, the governing equations (8) and (22) for the electrochemical potentials of the ions and the fluid flow field must be solved by satisfying the boundary conditions (10), (12), (13), and (23)-(25) with $c \rightarrow \infty$. The method-of-reflection solution consists of the series, whose terms depend on increasing powers of $\lambda$,

$\mu_{m}=\mu_{m \infty}+\mu_{m \mathrm{p}}^{(1)}+\mu_{m \mathrm{w}}^{(1)}+\mu_{m \mathrm{p}}^{(2)}+\mu_{m \mathrm{w}}^{(2)}+\cdots$,

$\mathbf{v}=\mathbf{v}_{\mathrm{p}}^{(1)}+\mathbf{v}_{\mathrm{w}}^{(1)}+\mathbf{v}_{\mathrm{p}}^{(2)}+\mathbf{v}_{\mathrm{w}}^{(2)}+\cdots$,

where subscripts $\mathrm{p}$ and $\mathrm{w}$ represent the reflections from the particle and the wall, respectively, and the superscript $(i)$ denotes the $i$ th reflection from that surface. In these series, all the expansion sets of the corresponding electrochemical potential and velocity fields for the fluid solution must satisfy Eqs. (8) and (22). The advantage of this method is that it is necessary to consider boundary conditions associated with only one surface at a time.

According to Eq. (A.1), the translational and angular velocities of the particle can also be expressed in the series forms

$\mathbf{U}=U_{0} \mathbf{e}_{x}+\mathbf{U}^{(1)}+\mathbf{U}^{(2)}+\cdots$,

$\boldsymbol{\Omega}=\boldsymbol{\Omega}^{(1)}+\boldsymbol{\Omega}^{(2)}+\cdots$.

In these expressions, $U_{0}$ is the diffusiophoretic velocity of an identical particle suspended freely in the continuous phase far from the wall given by Eq. (7); $\mathbf{U}^{(i)}$ and $\boldsymbol{\Omega}^{(i)}$ are related to $\nabla \mu_{m \mathrm{w}}^{(i)}$ and $\mathbf{v}_{\mathrm{w}}^{(i)}$ by [33]

$\mathbf{U}^{(i)}=\sum_{m=1}^{2} G_{m}\left[\nabla \mu_{m \mathrm{w}}^{(i)}\right]_{0}+\left[\mathbf{v}_{\mathrm{w}}^{(i)}\right]_{0}+\frac{a^{2}}{6}\left[\nabla^{2} \mathbf{v}_{\mathrm{w}}^{(i)}\right]_{0}$,

$\boldsymbol{\Omega}^{(i)}=\frac{1}{2}\left[\nabla \times \mathbf{v}_{\mathrm{w}}^{(i)}\right]_{0}$,

where the subscript 0 to variables inside brackets denotes evaluation at the position of the particle center. In Eq. (A.3a),

$$
\begin{aligned}
G_{1}= & \frac{\varepsilon k T}{3 \pi \eta(Z e)^{2}}\left[\left(1+g_{11}-g_{21}\right) \bar{\zeta}\right. \\
& \left.+\left(1+g_{11}+g_{21}\right) \ln \cosh \bar{\zeta}\right], \\
G_{2}= & \frac{\varepsilon k T}{3 \pi \eta(Z e)^{2}}\left[\left(-1+g_{12}-g_{22}\right) \bar{\zeta}\right. \\
& \left.+\left(1+g_{12}+g_{22}\right) \ln \cosh \bar{\zeta}\right],
\end{aligned}
$$

where $\bar{\zeta}$ is defined by Eq. (4),

$g_{11}=\frac{1}{2}\left(c_{1}^{\prime}+c_{1}\right)$,

$$
\begin{aligned}
& g_{12}=\frac{1}{2}\left(c_{1}^{\prime}-c_{1}\right), \\
& g_{21}=\frac{1}{2}\left(c_{2}^{\prime}-c_{2}\right), \\
& g_{22}=\frac{1}{2}\left(c_{2}^{\prime}+c_{2}\right) .
\end{aligned}
$$

In Eq. (A.5),

$$
\begin{aligned}
c_{1}= & \frac{1}{2 a^{2} \Delta_{1}}\left(a^{2}-2 a \beta_{11}+3 a \beta_{12}\right. \\
& \left.+a \beta_{22}+2 \beta_{12} \beta_{21}-2 \beta_{11} \beta_{22}\right), \\
c_{2}= & \frac{1}{2 a^{2} \Delta_{1}}\left(a^{2}-2 a \beta_{22}+3 a \beta_{21}+a \beta_{11}\right. \\
& \left.+2 \beta_{12} \beta_{21}-2 \beta_{11} \beta_{22}\right), \\
c_{1}^{\prime}= & c_{1}-3 \frac{\beta_{12}}{a \Delta_{1}}, \\
c_{2}^{\prime}= & c_{2}-3 \frac{\beta_{21}}{a \Delta_{1}},
\end{aligned}
$$

where

$\Delta_{1}=\frac{1}{a^{2}}\left(a^{2}+a \beta_{11}+a \beta_{22}-\beta_{12} \beta_{21}+\beta_{11} \beta_{22}\right)$,

and the relaxation coefficients $\beta_{11}, \beta_{12}, \beta_{21}$, and $\beta_{22}$ are defined by Eq. (11). In the limit of Eq. (6) or zero relaxation coefficients, Eqs. (A.5) and (A.6) reduce to $c_{1}=c_{2}=c_{1}^{\prime}=$ $c_{2}^{\prime}=g_{11}=g_{22}=1 / 2$ and $g_{12}=g_{21}=0$. In the other limit of very large relaxation coefficients, these equations become $c_{1}=c_{2}=c_{1}^{\prime}=c_{2}^{\prime}=g_{11}=g_{22}=-1$ and $g_{12}=g_{21}=0$.

The solution for the first reflected fields from the particle is

$\mu_{m \mathrm{p}}^{(1)}=a^{3} r^{-2} \sin \theta \cos \phi \sum_{i=1}^{2} g_{m i}\left|\nabla \mu_{i \infty}\right|$,

$\mathbf{v}_{\mathrm{p}}^{(1)}=\frac{1}{2} U_{0} a^{3} r^{-3}\left(2 \sin \theta \cos \phi \mathbf{e}_{r}\right.$

$$
\left.-\cos \theta \cos \phi \mathbf{e}_{\theta}+\sin \phi \mathbf{e}_{\phi}\right) \text {. }
$$

The velocity distribution shown in Eq. (A.8b) is identical to the irrotational flow surrounding a rigid sphere moving with velocity $U_{0} \mathbf{e}_{x}$.

The boundary conditions for the first reflected fields from the wall are derived from Eqs. (12), (13), (24), and (25):

$$
\begin{array}{r}
z=-b: \quad \frac{\partial \mu_{m \mathrm{w}}^{(1)}}{\partial z}=-\frac{\partial \mu_{m \mathrm{p}}^{(1)}}{\partial z}, \\
\mathbf{v}_{\mathrm{w}}^{(1)}=-\mathbf{v}_{\mathrm{p}}^{(1)} ; \\
r \rightarrow \infty, z>-b: \quad \begin{array}{l}
\mu_{m \mathrm{w}}^{(1)} \rightarrow 0 \\
\mathbf{v}_{\mathrm{w}}^{(1)} \rightarrow \mathbf{0} .
\end{array}
\end{array}
$$


The solution of $\mu_{m \mathrm{w}}^{(1)}$ is obtained by applying complex Fourier transforms on $x$ and $y$ in Eqs. (8) and (A.9a), (A.9c), with the result

$\mu_{m \mathrm{w}}^{(1)}=a^{3} x\left[x^{2}+y^{2}+(z+2 b)^{2}\right]^{-3 / 2} \sum_{i=1}^{2} g_{m i}\left|\nabla \mu_{i \infty}\right|$.

This reflected concentration field may be interpreted as arising from the reflection of the imposed field from a fictitious particle identical to the actual particle, its location being at the mirror-image position of the actual particle with respect to the plane $z=-b$ (i.e., at $x=0, y=0, z=-2 b$ ). The solution for $\mathbf{v}_{\mathrm{W}}^{(1)}$ can be found by fitting the boundary conditions (A.9b), (A.9d) with the general solution to Eq. (22) established by Faxen [34], which results in

$$
\begin{aligned}
\mathbf{v}_{\mathrm{w}}^{(1)}= & \frac{a^{3} U_{0}}{4 \pi} \int_{-\infty}^{\infty} \int_{-\infty}^{\infty} e^{i(\hat{\alpha} x+\hat{\beta} y)-\kappa(z+2 b)} \\
& \times\left\{-[2 \kappa(z+b)+1] i \hat{\alpha} \mathbf{e}_{z}-[2 \kappa(z+b)-1]\right. \\
& \left.\times\left(\frac{\hat{\alpha}^{2}}{\kappa} \mathbf{e}_{x}+\frac{\hat{\alpha} \hat{\beta}}{\kappa} \mathbf{e}_{y}\right)\right\} \mathrm{d} \hat{\alpha} \mathrm{d} \hat{\beta},
\end{aligned}
$$

where $\kappa=\left(\hat{\alpha}^{2}+\hat{\beta}^{2}\right)^{1 / 2}$ and $i=\sqrt{-1}$.

The contributions of $\mu_{m \mathrm{w}}^{(1)}$ and $\mathbf{v}_{\mathrm{w}}^{(1)}$ to the translational and angular velocities of the particle are determined by using Eq. (A.3),

$\mathbf{U}_{\mathrm{s}}^{(1)}=\sum_{m=1}^{2} G_{m}\left[\nabla \mu_{m \mathrm{w}}^{(1)}\right]_{r=0}=\frac{1}{8} G \lambda^{3} U_{0} \mathbf{e}_{x}$,

$\mathbf{U}_{\mathrm{h}}^{(1)}=\left[\mathbf{v}_{\mathrm{w}}^{(1)}+\frac{a^{2}}{6} \nabla^{2} \mathbf{v}_{\mathrm{w}}^{(1)}\right]_{r=0}=-\frac{1}{8}\left(\lambda^{3}-\lambda^{5}\right) U_{0} \mathbf{e}_{x}$,

$\mathbf{U}^{(1)}=\mathbf{U}_{\mathrm{s}}^{(1)}+\mathbf{U}_{\mathrm{h}}^{(1)}=\frac{1}{8}\left[-(1-G) \lambda^{3}+\lambda^{5}\right] U_{0} \mathbf{e}_{x}$,

$a \boldsymbol{\Omega}^{(1)}=\frac{a}{2}\left[\nabla \times \mathbf{v}_{\mathrm{w}}^{(1)}\right]_{r=0}=-\frac{3}{16} U_{0} \lambda^{4} \mathbf{e}_{y}$,

where

$G=\frac{1}{U_{0}} \sum_{m=1}^{2} \sum_{i=1}^{2} G_{m} g_{m i}\left|\nabla \mu_{i \infty}\right|$

After the substitution of Eq. (14) for $\mu_{i \infty}$ and Eq. (A.5) for $g_{m i}$, Eq. (A.12) becomes

$G=\frac{k T\left|\nabla n^{\infty}\right|}{U_{0} n^{\infty}(\mathbf{0})} \sum_{m=1}^{2} G_{m}\left[c_{m}^{\prime}-(-1)^{m} c_{m} \alpha\right]$.

Equation (A.11a) shows that the reflected electrochemical potential field from the wall can increase (if $G>0$ ) or decrease (if $G<0$ ) the velocity of the diffusiophoretic particle, while Eq. (A.11b) indicates that the reflected velocity field is to decrease this velocity; the net effect of the reflected fields is expressed by Eq. (A.11c), which can enhance or retard the movement of the particle, depending on the combination of the values of $G$ and $\lambda$. When $G=0$, the reflected electrochemical potential field makes no contribution to the diffusiophoretic velocity. Equation (A.11c) indicates that the wall correction to the translational velocity of the diffusiophoretic particle is $O\left(\lambda^{3}\right)$, which is weaker than that obtained for the corresponding sedimentation problem, in which the leading boundary effect is $O(\lambda)$. Note that the necessary condition for the wall enhancement on the diffusiophoretic motion to occur is a large value of $G$ and/or a value of $\lambda$ close to unity such that the relation $\lambda^{5}>(1-G) \lambda^{3}$ is warranted.

Equation (A.11d) shows that the diffusiophoretic sphere rotates about an axis which is perpendicular to the direction of the applied electrolyte concentration gradient and parallel to the plane wall. The direction of rotation is opposite to that which would occur if the sphere were driven to move by a body force. Note that the angular velocity $\boldsymbol{\Omega}^{(1)}$ in Eq. (A.11d) does not depend on the parameter $G$ (since $\mathbf{v}_{\mathrm{W}}^{(1)}$ is not a function of $G$ ). Also, the wall-induced angular velocity of the diffusiophoretic particle is $O\left(\lambda^{4}\right)$, which is the same in order as but different in its coefficient $(-3 / 16$ versus $3 / 32$ ) from that of a rigid sphere moving under a body-force field [34].

$\mathbf{U}^{(2)}$ and $a \mathbf{\Omega}^{(2)}$ will be $O\left(\lambda^{6}\right) \mathbf{e}_{x}$ and $O\left(\lambda^{7}\right) \mathbf{e}_{y}$, respectively. With the substitution of Eqs. (A.11c) and (A.11d) into Eq. (A.2), the particle velocities can be expressed as $\mathbf{U}=U \mathbf{e}_{x}$ and $\boldsymbol{\Omega}=\Omega \mathbf{e}_{y}$ with

$$
\begin{aligned}
& U=U_{0}\left[1-\frac{1}{8}(1-G) \lambda^{3}+\frac{1}{8} \lambda^{5}+O\left(\lambda^{6}\right)\right], \\
& a \Omega=U_{0}\left[-\frac{3}{16} \lambda^{4}+O\left(\lambda^{7}\right)\right] .
\end{aligned}
$$

The particle migrates along the imposed electrolyte concentration gradient at a rate that can increase or decrease as the particle approaches the wall. Owing to the neglect of inertial effects, the wall does not deflect the direction of diffusiophoresis.

For the case that a linear electrolyte concentration profile is prescribed on the plane wall which is consistent with the far-field distribution, namely, the boundary condition (12) is replaced by Eq. (15), the series expansions (A.1) and (A.2), the solutions of $\mu_{m p}^{(1)}$ and $\mathbf{v}_{\mathrm{p}}^{(1)}$ in Eq. (A.8), and the boundary conditions for $\mu_{m \mathrm{w}}^{(1)}$ and $\mathbf{v}_{\mathrm{w}}^{(1)}$ in Eqs. (A.9b)-(A.9d) are still valid, while Eq. (A.9a) becomes

$z=-b: \quad \mu_{m \mathrm{w}}^{(1)}=-\mu_{m \mathrm{p}}^{(1)}$.

With this change, it can be shown that the results of the reflected fields and of the particle velocities are also obtained from Eqs. (A.10)-(A.14) by replacing $g_{m i}$ by $-g_{m i}$ (or replacing $G$ by $-G$ ). Thus, contrary to the effect of an impermeable and nonconducting plane wall, the reflected electrochemical potential field from a parallel wall with the 
imposed far-field concentration gradient reduces the translational velocity of the particle if $G>0$ and enhances this velocity if $G<0$. When $G=0$, the two types of plane wall will produce the same effects on the diffusiophoretic motion of the particle. Under the condition that the value of $G$ is sufficiently small and/or the value of $\lambda$ is sufficiently large such that $\lambda^{5}>(1+G) \lambda^{3}$, the net effect of a lateral plane wall prescribed with the far-field concentration distribution can enhance the diffusiophoretic migration of a particle.

For the problem of electrophoretic motion of a spherical particle caused by a constant external electric field $\mathbf{E}^{\infty}$ parallel to a nonconducting plane or to a plane prescribed with a linear electric potential profile consistent with the far-field distribution, the above analysis still applies, but now $U_{0}$ is the electrophoretic velocity of an isolated sphere given by Eq. (5) and the expression for $G$ given by Eq. (A.13) becomes

$G=-\frac{Z e\left|\mathbf{E}^{\infty}\right|}{U_{0}} \sum_{m=1}^{2}(-1)^{m} G_{m} c_{m}$.

Note that Eq. (A.14b) for the rotational velocity of the particle correct to $O\left(\lambda^{4}\right)$ is independent of the parameter $G$.

\section{A.2. Motion on the median plane between two parallel flat walls}

For the problem of diffusiophoretic or electrophoretic motion of a sphere on the median plane between two impermeable and nonconducting parallel plates, the boundary conditions corresponding to governing equations (8) and (22) are given by Eqs. (10), (12), (13), and (23)-(25) with $c=b$. But, the angular velocity $\boldsymbol{\Omega}$ of the particle vanishes now because of the symmetry. With $\lambda=a / b \ll 1$, the series expansions of the electrochemical potentials of the ions, fluid velocity, and particle velocity given by Eqs. (A.1), (A.2a), and (A.8) remain valid here. From Eqs. (12), (13), (24), and (25), the boundary conditions for the first wall-reflected fields $\mu_{m \mathrm{w}}^{(1)}$ and $\mathbf{v}_{\mathrm{w}}^{(1)}$ are found to be

$$
\begin{aligned}
&|z|=b: \quad \frac{\partial \mu_{m \mathrm{w}}^{(1)}}{\partial z}=-\frac{\partial \mu_{m \mathrm{p}}^{(1)}}{\partial z}, \\
& \mathbf{v}_{\mathrm{w}}^{(1)}=- \mathbf{v}_{\mathrm{p}}^{(1)} ; \\
& r \rightarrow \infty,|z| \leqslant b: \quad \mu_{m \mathrm{w}}^{(1)} \rightarrow 0, \\
& \quad \mathbf{v}_{\mathrm{w}}^{(1)} \rightarrow \mathbf{0} .
\end{aligned}
$$

The first wall-reflected fields can be solved by the same method as used for a single lateral plate in the previous section, with the results

$$
\begin{aligned}
\mu_{m \mathrm{w}}^{(1)}= & -\frac{a^{3}}{2 \pi} \int_{-\infty}^{\infty} \int_{-\infty}^{\infty} \frac{i \alpha}{\kappa} e^{i(\hat{\alpha} x+\hat{\beta} y)-\kappa b} \frac{\cosh (\kappa z)}{\sinh (\kappa b)} \mathrm{d} \hat{\alpha} \mathrm{d} \hat{\beta} \\
& \times \sum_{i=1}^{2} g_{m i}\left|\nabla \mu_{i \infty}\right|,
\end{aligned}
$$

$$
\begin{aligned}
\mathbf{v}_{\mathrm{w}}^{(1)}= & \frac{a^{3}}{2 \pi} U_{0} \int_{-\infty}^{\infty} \int_{-\infty}^{\infty} \frac{1}{\sinh (2 \kappa b)-2 \kappa b} e^{i(\hat{\alpha} x+\hat{\beta} y)} \\
& \times\left\{[\sinh (\kappa z)-\kappa z \cosh (\kappa z)+g \sinh (\kappa z)] i \hat{\alpha} \mathbf{e}_{z}\right. \\
& +[\kappa z \sinh (\kappa z)-g \cosh (\kappa z)] \\
& \left.\times\left(\frac{\hat{\alpha}^{2}}{\kappa} \mathbf{e}_{x}+\frac{\hat{\alpha} \hat{\beta}}{\kappa} \mathbf{e}_{y}\right)\right\} \mathrm{d} \hat{\alpha} \mathrm{d} \hat{\beta},
\end{aligned}
$$

where $\kappa=\left(\hat{\alpha}^{2}+\hat{\beta}^{2}\right)^{1 / 2}, g=\kappa b-e^{-\kappa b} \sinh (\kappa b)$, and $U_{0}$ is given by Eqs. (7) and (5) for the cases of diffusiophoresis and electrophoresis, respectively. The contributions of $\mu_{m \mathrm{w}}^{(1)}$ and $\mathbf{v}_{\mathrm{W}}^{(1)}$ to the particle velocity are determined using Eq. (A.3a), which lead to a result similar to Eqs. (A.11a)-(A.11c),

$$
\begin{aligned}
\mathbf{U}_{\mathrm{s}}^{(1)} & =d_{1} G \lambda^{3} U_{0} \mathbf{e}_{x}, \\
\mathbf{U}_{\mathrm{h}}^{(1)} & =-\left(d_{2} \lambda^{3}-d_{3} \lambda^{5}\right) U_{0} \mathbf{e}_{x}, \\
\mathbf{U}^{(1)} & =\mathbf{U}_{\mathrm{s}}^{(1)}+\mathbf{U}_{\mathrm{h}}^{(1)} \\
& =\left[-\left(d_{2}-d_{1} G\right) \lambda^{3}-d_{3} \lambda^{5}\right] U_{0} \mathbf{e}_{x},
\end{aligned}
$$

where $G$ is given by Eq. (A.12) (or by Eqs. (A.13) and (A.16) for the relevant cases),

$$
\begin{aligned}
& d_{1}=\int_{0}^{\infty} \frac{\rho^{2}}{e^{2 \rho}-1} \mathrm{~d} \rho=0.300514, \\
& d_{2}=\frac{1}{2} \int_{0}^{\infty} \frac{\rho^{2}\left(\rho-e^{-\rho} \sinh \rho\right)}{\sinh (2 \rho)-2 \rho} \mathrm{d} \rho=0.417956, \\
& d_{3}=\frac{1}{6} \int_{0}^{\infty} \frac{\rho^{4}}{\sinh (2 \rho)-2 \rho} \mathrm{d} \rho=0.338324 .
\end{aligned}
$$

With the combination of Eqs. (A.2a) and (A.19c), the particle velocity can be expressed as $\mathbf{U}=U \mathbf{e}_{x}$ with

$$
U=U_{0}\left[1-\left(d_{2}-d_{1} G\right) \lambda^{3}+d_{3} \lambda^{5}+O\left(\lambda^{6}\right)\right] .
$$

For the case where the particle is undergoing diffusiophoresis or electrophoresis on the median plane between two parallel plates on which a linear electrolyte concentration or electric potential profile consistent with the far-field distribution is imposed, Eq. (12) should be replaced by Eq. (15). In this case, Eqs. (A.1), (A.2a), (A.8), (A.17b)-(A.17d) are still applicable, while Eq. (A.17a) becomes

$|z|=b: \quad \mu_{m \mathrm{w}}^{(1)}=-\mu_{m \mathrm{p}}^{(1)}$.

With this change, it can be shown that the results of the reflected fields and of the particle velocity are also obtained from Eqs. (A.18)-(A.21) by replacing $g_{m i}$ (or $G$ ) and $d_{1}$ by 
$-g_{m i}($ or $-G)$ and $\bar{d}_{1}$, respectively, where

$\bar{d}_{1}=\int_{0}^{\infty} \frac{\rho^{2}}{e^{2 \rho}+1} \mathrm{~d} \rho=0.225386$.

Comparing Eq. (A.21) for the slit case with Eq. (A.14a) for the case of a single parallel plane, one can find that the wall effects on the diffusiophoretic or electrophoretic velocity of a particle in the two cases are qualitatively similar. However, the assumption that the result of the boundary effect for two walls can be obtained by simple addition of the single-wall effects generally gives a smaller correction to diffusiophoretic or electrophoretic velocity, while for the corresponding sedimentation problem this approximation overestimates the wall correction.

Using the same method of reflections, Keh and Anderson [23] obtained asymptotic solutions for the electrophoretic mobility of a dielectric sphere surrounded by an infinitesimally thin electric double layer either parallel to a nonconducting plane wall or on the median plane between two parallel plates correct to $O\left(\lambda^{6}\right)$. In the limit of Eq. (6), the formulas given by Eqs. (A.14a) and (A.21) are consistent with these earlier solutions.

As discussed in Sections 3 and 4, the boundary effects on phoretic motions in general are quite complicated and no simple rule is able to make an adequate prediction for such complicated phenomena. Thus, limited numerical solutions with interpolation and extrapolation are awkward to be used in practical applications. Therefore, the closed-form analytical results obtained in this Appendix, which can be conveniently used in the calculations for various cases with $\kappa a>20$ (without the need of a computer), should be a favorable contribution to the evaluation and understanding of the boundary effects on diffusiophoresis and electrophoresis.

\section{Appendix B. Definitions of some functions in Section 2}

The functions $\delta_{n}^{(i)}$ with $i=1,2,3$, and 4 in Eqs. (19), (21), (31), and (36) are defined by

$$
\begin{aligned}
\delta_{n}^{(1)}(r, \mu)= & r^{-n-1} P_{n}^{1}(\mu)-(-n)^{m} \int_{0}^{\infty} \kappa^{1-m} \frac{J_{1}(\kappa \rho)}{\sinh \tau} \\
& \times\left[c^{2} V_{n+m}(c)(\sinh \sigma)^{1-m}(\cosh \sigma)^{m}\right. \\
& \left.-b^{2} V_{n+m}(-b)(\sinh \omega)^{1-m}(\cosh \omega)^{m}\right] \mathrm{d} \kappa,
\end{aligned}
$$

$$
\begin{aligned}
\delta_{n}^{(2)}(r, \mu)= & -(n+1) r^{-n-2} P_{n}^{1}(\mu) \\
& -(-n)^{m} \int_{0}^{\infty} \frac{\kappa^{2-m}}{\sinh \tau}\left\{J_{1}^{\prime}(\kappa \rho)\left(1-\mu^{2}\right)^{1 / 2}\right. \\
& \times\left[c^{2} V_{n+m}(c)(\sinh \sigma)^{1-m}(\cosh \sigma)^{m}\right. \\
& \left.-b^{2} V_{n+m}(-b)(\sinh \omega)^{1-m}(\cosh \omega)^{m}\right]
\end{aligned}
$$

$$
\begin{aligned}
& +J_{1}(\kappa \rho) \mu\left[c^{2} V_{n+m}(c)(\cosh \sigma)^{1-m}(\sinh \sigma)^{m}\right. \\
& \left.\left.-b^{2} V_{n+m}(-b)(\cosh \omega)^{1-m}(\sinh \omega)^{m}\right]\right\} \mathrm{d} \kappa,
\end{aligned}
$$

$$
\begin{aligned}
\delta_{n}^{(3)}(r, \mu)= & -r^{-n-1} \frac{\partial P_{n}^{1}(\mu)}{\partial \mu}\left(1-\mu^{2}\right)^{1 / 2} \\
& -(-n)^{m} r \int_{0}^{\infty} \frac{\kappa^{2-m}}{\sinh \tau}\left\{J_{1}^{\prime}(\kappa \rho) \mu\right. \\
& \times\left[c^{2} V_{n+m}(c)(\sinh \sigma)^{1-m}(\cosh \sigma)^{m}\right. \\
& \left.-b^{2} V_{n+m}(-b)(\sinh \omega)^{1-m}(\cosh \omega)^{m}\right] \\
& -J_{1}(\kappa \rho)\left(1-\mu^{2}\right)^{1 / 2} \\
& \times\left[c^{2} V_{n+m}(c)(\cosh \sigma)^{1-m}(\sinh \sigma)^{m}\right. \\
& \left.\left.-b^{2} V_{n+m}(-b)(\cosh \omega)^{1-m}(\sinh \omega)^{m}\right]\right\} \mathrm{d} \kappa,
\end{aligned}
$$

$$
\begin{aligned}
\delta_{n}^{(4)}(r, \mu)= & (n+1)(n+2) r^{-n-3} P_{n}^{1}(\mu) \\
& -(-n)^{m} \int_{0}^{\infty} \frac{\kappa^{3-m}}{\sinh \tau}\left\{\left[J_{1}^{\prime \prime}(\kappa \rho)\left(1-\mu^{2}\right)\right.\right. \\
& \left.+J_{1}(\kappa \rho) \mu^{2}\right]\left[-c^{2} V_{n+m}(\sinh \sigma)^{1-m}(\cosh \sigma)^{m}\right. \\
& \left.-b^{2} V_{n+m}(-b)(\sinh \omega)^{1-m}(\cosh \omega)^{m}\right] \\
& +2 J_{1}^{\prime}(\kappa \rho)\left(1-\mu^{2}\right)^{1 / 2} \mu \\
& \times\left[c^{2} V_{n+m}(c)(\cosh \sigma)^{1-m}(\sinh \sigma)^{m}\right. \\
& \left.\left.-b^{2} V_{n+m}(-b)(\cosh \omega)^{1-m}(\sinh \omega)^{m}\right]\right\} \mathrm{d} \kappa .
\end{aligned}
$$

Here,

$$
\begin{aligned}
V_{n}\left(z_{i}\right)= & \frac{(2 / \pi)^{1 / 2}}{z_{i}^{n+1}} \sum_{q=0}^{[n / 2]} \frac{\left(\kappa\left|z_{i}\right|\right)^{n-q-1 / 2}}{(-2)^{q} q !(n-2 q-1) !} \\
& \times K_{n-q-3 / 2}\left(\kappa\left|z_{i}\right|\right),
\end{aligned}
$$

$$
\begin{aligned}
& \sigma=\kappa(z+b), \\
& \omega=\kappa(z-c), \\
& \tau=\kappa(b+c),
\end{aligned}
$$

$J_{1}$ is the Bessel function of the first kind of order one and the prime on it denotes differentiation with respect to its argument, $K_{v}$ is the modified Bessel function of the second kind of order $v$, and the square bracket $[v]$ denotes the largest integer which is less than or equal to $v$. In Eqs. (B.1)-(B.4), $m=1$ if Eq. (12) is used for the boundary condition of the electrochemical potential field at the plane walls and $m=0$ if Eq. (15) is used. 


\section{References}

[1] S.S. Dukhin, B.V. Derjaguin, in: E. Matijevic (Ed.), Surface and Colloid Science, vol. 7, Wiley, New York, 1974.

[2] J.L. Anderson, Annu. Rev. Fluid Mech. 21 (1989) 61.

[3] A. Acrivos, D.J. Jeffrey, D.A. Saville, J. Fluid Mech. 212 (1990) 95.

[4] H.J. Keh, S.B. Chen, Langmuir 9 (1993) 1142.

[5] R.J. Hunter, Zeta Potential in Colloidal Science, Academic Press, London, 1981.

[6] W.B. Russel, D.A. Saville, W.R. Schowalter, Colloidal Dispersions, Cambridge Univ. Press, Cambridge, 1989.

[7] S.P. Radko, A. Chrambach, Electrophoresis 23 (2002) 1957.

[8] D.C. Henry, Proc. R. Soc. London A 133 (1931) 106.

[9] F.A. Morrison, J. Colloid Interface Sci. 34 (1970) 210

[10] J.H. Masliyah, Electrokinetic Transport Phenomena, AOSTRA, Edmonton, 1994.

[11] R.E. Smith, D.C. Prieve, Chem. Eng. Sci. 37 (1982) 1213.

[12] D.C. Prieve, Adv. Colloid Interface Sci. 16 (1982) 321.

[13] D.C. Prieve, J.L. Anderson, J.P. Ebel, M.E. Lowell, J. Fluid Mech.148 (1984) 247.

[14] R.W. O’Brien, L.R. White, J. Chem. Soc. Faraday Trans. 274 (1978) 1607.

[15] R.W. O’Brien, J. Colloid Interface Sci. 92 (1983) 204.
[16] S.B. Chen, H.J. Keh, J. Fluid Mech. 238 (1992) 251.

[17] D.C. Prieve, R. Roman, J. Chem. Soc. Faraday Trans. 283 (1987) 1287.

[18] Y. Pawar, Y.E. Solomentsev, J.L. Anderson, J. Colloid Interface Sci. 155 (1993) 488.

[19] H.A. Stone, S. Kim, AIChE J. 47 (2001) 1250.

[20] H.A. Stone, A.D. Stroock, A. Ajdari, Annu. Rev. Fluid Mech. 36 (2004) 381

[21] C. Ye, D. Li, Microfluid Nanofluid 1 (2004) 52.

[22] H. Liu, H.H. Bau, H.H. Hu, Langmuir 20 (2004) 2628.

[23] H.J. Keh, J.L. Anderson, J. Fluid Mech. 153 (1985) 417.

[24] H.J. Keh, S.B. Chen, J. Fluid Mech. 194 (1988) 377.

[25] H.J. Keh, L.C. Lien, J. Fluid Mech. 224 (1991) 305.

[26] M. Loewenberg, R.H. Davis, J. Fluid Mech. 288 (1995) 103.

[27] H.J. Keh, J.Y. Chiou, AIChE J. 42 (1996) 1397.

[28] E. Yariv, H. Brenner, Phys. Fluids 14 (2002) 3354.

[29] E. Yariv, H. Brenner, J. Fluid Mech. 484 (2003) 85.

[30] H.J. Keh, J.S. Jan, J. Colloid Interface Sci. 183 (1996) 458.

[31] V.G. Levich, Physicochemical Hydrodynamics, Prentice Hall, Englewood Cliffs, NJ, 1962.

[32] P. Ganatos, S. Weinbaum, R. Pfeffer, J. Fluid Mech. 99 (1980) 755.

[33] H.J. Tu, H.J. Keh, J. Colloid Interface Sci. 231 (2000) 265.

[34] J. Happel, H. Brenner, Low Reynolds Number Hydrodynamics, Nijhoff, 1983. 